\title{
Database analysis of errors in large-eddy simulation
}

\author{
Johan Meyers ${ }^{\mathrm{a})}$ \\ Department of Mechanical Engineering, Katholieke Universiteit Leuven, Celestijnenlaan 300, \\ B3001 Leuven, Belgium \\ Bernard J. Geurts ${ }^{\text {b) }}$ \\ Mathematical Sciences, J.M. Burgers Center, University of Twente, P.O. Box 217, 7500 AE Enschede, \\ The Netherlands \\ Martine Baelmans ${ }^{\mathrm{c}}$ \\ Department of Mechanical Engineering, Katholieke Universiteit Leuven, Celestijnenlaan 300, \\ B3001 Leuven, Belgium
}

(Received 10 January 2003; accepted 12 June 2003; published 5 August 2003)

\begin{abstract}
A database of decaying homogeneous, isotropic turbulence is constructed including reference direct numerical simulations at two different Reynolds numbers and a large number of corresponding large-eddy simulations at various subgrid resolutions. Errors in large-eddy simulation as a function of physical and numerical parameters are investigated. In particular, employing the Smagorinsky subgrid parametrization, the dependence of modeling and numerical errors on simulation parameters is quantified. The interaction between these two basic sources of error is shown to lead to their partial cancellation for several flow properties. This leads to a central paradox in large-eddy simulation related to possible strategies that can be followed to improve the accuracy of predictions. Moreover, a framework is presented in which the global parameter dependence of the errors can be classified in terms of the "subgrid activity" which measures the ratio of the turbulent to the total dissipation rate. Such an analysis allows one to quantify refinement strategies and associated model parameters which provide optimal total simulation error at given computational cost. (c) 2003 American Institute of Physics. [DOI: 10.1063/1.1597683]
\end{abstract}

\section{INTRODUCTION}

As an integral part of the development of large-eddy simulation (LES), the closure of the filtered Navier-Stokes equations has arisen as an important issue. Kolmogorov's theory (translated, e.g., in Ref. 1) indicates a universal behavior of the "small scales" in high Reynolds number turbulence. In this context LES is considered a promising approach to treat high Reynolds number flows. Indeed, LES explicitly calculates the large flow-specific turbulent eddies and adopts a so-called subgrid-scale (sgs) model to represent the dynamic effects of the universal small-scale flow features which are not resolved in the simulation. However, next to subgrid modeling, effects arising from inadequate subgrid resolution constitute an equally important element in the development of LES. In addition, the various sources of error can interact, which may lead to further complications in the interpretation of LES. In this paper we analyze the total interacting error dynamics through a judicious combination of simulations at appropriately chosen numerical and physical parameters.

Research in the past three decades revealed that the development of the basic LES philosophy in actual flow applications is not straightforward. Witness to this is, e.g., the lack of a universal subgrid-scale model. The existing models can

\footnotetext{
${ }^{a)}$ Electronic mail: Johan.Meyers@mech.kuleuven.ac.be

${ }^{b)}$ Electronic mail: B.J.Geurts@math.utwente.nl

${ }^{c)}$ Electronic mail: Martine.Baelmans@mech.kuleuven.ac.be
}

be roughly grouped into a number of basic categories including eddy-viscosity models, ${ }^{2-5}$ stochastic models, ${ }^{6,7}$ similarity models, ${ }^{8-11}$ mixed models, ${ }^{12-16}$ inverse modeling, ${ }^{17-19}$ and, quite recently, assumed sgs velocity models $\mathrm{s}^{20-23}$ and regularization models. ${ }^{24}$ In addition, an important development in subgrid scale modeling arose with the dynamic procedure $^{25-27}$ which allows optimization of parameters in subgrid models in accordance with the turbulent flow that is simulated. This leads to an explicit determination of model coefficients without introducing external ad hoc parameters.

Although several of these models give good results in various specific simulations, the quality of actual LES predictions in general depends on a large number of additional factors that are part of the computational modeling of a flow. In fact, complications may arise from the specific flow geometry, from the flow regime, e.g., specified by the value of the Reynolds number, from the mesh and mesh size, and from the numerical method. All these factors can simultaneously be quite important in view of the marginal resolution of many dynamically significant features of the flow. This marginal resolution is imposed by practical restrictions arising from the computational capabilities of present-day computers.

The variety of elements that constitute a complete numerical large-eddy modeling of a turbulent flow may lead to an intricate, nonlinear interaction among the modeling and numerical errors. Indeed, in many simulations to date, the magnitude of numerical errors can be in the range of the 
magnitude of the subgrid-scale stresses. ${ }^{28}$ In this "implicit modeling" by numerical errors one relies heavily on the properties of the particular spatial discretization method in relation to the smoothing of the flow. This introduces a considerable uncertainty in the predictions, e.g., when refining the computational grid. In an actual LES of a temporal mixing layer, Vreman et al. ${ }^{29}$ quantified the modeling and discretization errors for various flow properties. In this analysis, modeling and numerical errors were found to be of comparable magnitude and could partially cancel each other. This gives rise to some counter-intuitive effects related to improvements of LES predictions that might be achieved by adopting improved subgrid models and/or numerical methods. In this context, further error analysis for LES was presented in Refs. 30 and 31 where a clear error-pattern was observed in terms of the so-called "subgrid-activity" parameter $s$, which measures the relative subgrid-model dissipation rate. Hence $s$ quantifies the dynamic relevance of the small unresolved scales in a simulation. Roughly speaking, $s$ characterizes the "distance" between a direct numerical simulation (DNS) resolving all flow features at sufficiently high spatial resolution and an actual LES that corresponds to a specific filter width and mesh spacing.

Though, as mentioned earlier, various studies on simulation error exist, a practical systematic simulation framework in which the error analysis can be interpreted is still lacking. It is the aim of this paper to introduce a database approach for such an error analysis, where important parameters, such as the filter-width to mesh-size ratio, the integral length scale, the Reynolds number, etc., are included. To this end, decaying, homogeneous isotropic turbulence is considered at two different initial Taylor Reynolds numbers. For these flows reference DNS data are generated next to a corresponding set of LES based on the use of the Smagorinsky subgrid model. The database approach followed in the present paper complements the theoretical work by Ghosal (for an overview see Ref. 32) and provides an "experimental" approach to quantify the errors that arise in LES of homogeneous turbulence. In this approach the interactions between flow features of various length scales in the modeled equations are fully taken into account as well as the interaction between numerical and modeling errors. In particular, the database approach allows for a detailed decomposition of the total error effect in a numerical and a subgrid modeling contribution. This clarifies questions related to the dominant limitations of the accuracy of LES and clearly indicates in what way further improvements can be achieved. From such extensive comparative studies one may expect to distill a priori guidelines for the development of LES that meet pre-specified quality criteria.

The organization of this paper is as follows. First, in Sec. II, the results of the reference DNS are discussed with attention to the proper convergence of the solution field as a function of spatial resolution. Moreover, we specify the largeeddy formulation that is adopted. Next, in Sec. III the precise definition of the simulation error is provided and we concentrate on the temporal development of the total error and its main contributions. It is shown that the various "error components" can have effects with opposite sign and conse- quently may lead to simulations that are more accurate than could be expected from the individual error components. This leads to some interesting paradoxes which are discussed in more detail. Subsequently, in Sec. IV global error behavior is characterized in terms of the subgrid activity as proposed by Ref. 31 for different Reynolds numbers. This allows one to distinguish between different LES operating regions with respect to error behavior. Section V concentrates on the dependence of the total error on important simulation parameters such as spatial resolution and flow smoothing, e.g., through the specification of the filter width. A framework for visualizing these error dependencies is presented from which optimal simulation strategies can be inferred, e.g., concentrating on simulation error and/or computational effort. Finally, we summarize our findings in Sec. VI.

\section{REFERENCE SIMULATIONS AND LARGE-EDDY APPROACH}

In the following we first define the numerical model used to simulate decaying homogeneous isotropic turbulence and present a grid-refinement study establishing the accuracy of the DNS in Sec. II A. Then, in Sec. II B we show predictions of some basic flow properties such as the decay of the kinetic energy, the Taylor length-scale evolution and the skewness, which forms a point of reference for the error analysis in this paper. Finally, in Sec. II C we introduce the LES approach and specify the simulation settings.

\section{A. Direct numerical simulation: Numerical model and grid refinement}

In the direct numerical simulation approach the NavierStokes equations are solved on a sufficiently fine mesh which is adequate for resolving all relevant flow features. Incompressible flow is governed by

$$
\begin{aligned}
& \partial_{j} u_{j}=0, \\
& \partial_{t} u_{i}+\partial_{j}\left(u_{i} u_{j}\right)+\partial_{i} p-\frac{1}{\operatorname{Re}} \partial_{j j} u_{i}=0 ; \quad i=1,2,3
\end{aligned}
$$

where $u_{i}$ denotes the velocity in the $x_{i}$ direction and $p$ is the pressure. The summation convention for repeated indices is adopted and $\partial_{t}\left(\partial_{j}\right)$ denotes the partial derivative with respect to time $t$ (coordinate $x_{j}$ ). Moreover, Re is the computational Reynolds number based on the length of a side of the cubical flow domain. We adopt a physical space formulation in which the convective fluxes are discretized using a fourthorder accurate finite volume discretization ${ }^{33}$ while either a second- or a fourth-order accurate finite volume method is adopted for the viscous fluxes. ${ }^{34}$ In fact, the second-order scheme is used for the lower Reynolds number flow while a fourth-order scheme was found more suitable at the higher Reynolds number as will be discussed further. The time integration is done with an explicit four-stage compact storage Runge-Kutta scheme of second-order accuracy. The time steps are bounded by a stability condition. The grid used in the simulations is uniform with $n^{3}$ grid cells. Periodic conditions are enforced in all three coordinate directions. 
The reference decaying homogeneous isotropic turbulence is simulated at two different computational Reynolds numbers. These simulations can be characterized by different initial Taylor Reynolds numbers $\operatorname{Re}_{\lambda}$ for which we selected $\operatorname{Re}_{\lambda}=50$ and $\operatorname{Re}_{\lambda}=100$. The Taylor Reynolds number is defined as $\operatorname{Re}_{\lambda}=u^{\prime} \lambda / \nu$, where $u^{\prime}$ is the rms value of the velocity, $\nu$ is the kinematic viscosity, and $\lambda$ denotes the transverse Taylor length scale, which for homogeneous isotropic turbulence is defined by

$$
\frac{1}{\lambda^{2}}=\frac{1}{2 u^{\prime 2}}\left\langle\left(\frac{\partial u_{1}}{\partial x_{2}}\right)^{2}\right\rangle=\frac{1}{u^{\prime 2}}\left\langle\left(\frac{\partial u_{1}}{\partial x_{1}}\right)^{2}\right\rangle .
$$

The corresponding values of the computational Reynolds number $R e$ are on the order of 1061 and 4243 for $\operatorname{Re}_{\lambda}=50$, 100 , respectively.

The initial condition for the DNS is constructed based on a prescribed initial energy spectrum $E(k)$. The procedure to generate the initial state proceeds in three steps.

(1) A velocity field is generated in Fourier space with components $u_{i}(\mathbf{k})$. The phases of the components are random and taken from a uniform distribution. The amplitude of the components is taken equal to one at this stage.

(2) The solenoidal part of this field is calculated using $u_{i}^{s}(\mathbf{k})=P_{i j} u_{i}(\mathbf{k})$, with

$P_{i j}=\delta_{i j}+\frac{k_{i} k_{j}}{|\mathbf{k}|}$

a projection operator, which provides the perpendicular projection of $\mathbf{u}$ on $\mathbf{k}$.

(3) Finally, the Fourier amplitudes of the solenoidal field are rescaled such that the resulting spectrum corresponds to the prescribed $E(k)$.

The initial energy spectrum $E(k)$ is prescribed using an empirical expression which shows good resemblance with turbulent spectra of several types of flows. ${ }^{35}$ In detail, $E(k)$ is given by

$$
E(k)=\alpha \varepsilon^{2 / 3} k^{-5 / 3} f_{L}(k L) f_{\eta}\left(k L \operatorname{Re}_{L}^{-3 / 4}\right) .
$$

Here, $\alpha$ is the universal Kolmogorov constant (with value $\approx 1.5$ ) and $\varepsilon$ the mean energy dissipation. Moreover, $f_{L}$ and $f_{\eta}$ are further defined as

$$
\begin{aligned}
& f_{L}(z)=\left(\frac{z}{\left[z^{2}+c_{1}\right]^{1 / 2}}\right)^{11 / 3}, \\
& f_{\eta}(z)=\exp \left(-c_{2}\left(\left[z^{4}+c_{3}^{4}\right]^{1 / 4}-c_{3}\right)\right) .
\end{aligned}
$$

This spectrum is constructed consistent with a $k^{-5 / 3}$ spectrum. It is adapted in the low wavenumber range by a damping function $f_{L}(k L)$ and in the high wavenumber range by $f_{\eta}\left(k L \operatorname{Re}_{L}^{-3 / 4}\right)$. In fact, $f_{L}(k L)$ adapts the spectrum toward a Von Karman spectrum, which is characteristic for low wavenumber behavior of homogeneous isotropic turbulence. The integral length scale, $L$, used in this function is formally defined as

$$
L=\frac{\mathcal{E}^{3 / 2}}{\varepsilon},
$$

where $\mathcal{E}$ denotes the total turbulent kinetic energy contained in the solution, i.e., the appropriate integral of $E(k)$ over $k$. Further, $f_{\eta}\left(k L \mathrm{Re}_{L}^{-3 / 4}\right)$ adapts the spectrum to an exponential decay beyond the inertial subrange. The Reynolds number $\mathrm{Re}_{L}$ used here is defined as

$$
\operatorname{Re}_{L}=\frac{\mathcal{E}^{1 / 2} L}{\nu} .
$$

Its corresponding values are 375 and 1500 for $\operatorname{Re}_{\lambda}$ $=50,100$, respectively.

In the empirical expression for $E(k)$, three parameters $c_{1}, c_{2}$, and $c_{3}$ remain. They can be determined based on three consistency relations. First, $E(k)$ integrated over all wavenumbers should add up to the total kinetic energy. Further, the integration of $k^{2} E(k)$ should correspond to a correct level of total enstrophy. Analogously, $\int k^{4} E(k) d k$ should correspond to the correct palinstrophy level. An elaboration of these relations allows one to express $c_{1}, c_{2}$, and $c_{3}$ in terms of the Reynolds number $\mathrm{Re}_{L}$, the Kolmogorov constant $\alpha$, and the "formal" skewness of the longitudinal velocity derivative. The skewness is defined by

$$
S_{3}=\left\langle\left(\frac{\partial u_{1}}{\partial x_{1}}\right)^{3}\right\rangle /\left\langle\left(\frac{\partial u_{1}}{\partial x_{1}}\right)^{2}\right\rangle^{3 / 2} \text {. }
$$

For $S_{3}$, an initial value of -0.7 is adopted. Since the initial field is generated with random phases for the Fourier components the actual skewness according to Eq. (8) will be zero; hence the parameter $S_{3}=-0.7$ is only a formal parameter.

Finally, the reference length used in these simulations is the computational box size $\mathcal{L}$. For both initial Taylor Reynolds numbers the ratio of the box size to the integral length scale $\mathcal{L} / L$ is 2 . Further the initial energy level is 0.5 . This indicates that the viscosity at $\operatorname{Re}_{\lambda}=50$ is four times the viscosity in the $\operatorname{Re}_{\lambda}=100$ simulation.

The direct numerical simulations at both Reynolds numbers are performed using a compressible Navier-Stokes solver. The Mach number is set to a low value of 0.2 , which assures that the flow is nearly incompressible. This provides a representation of homogeneous turbulence which is suitable for our purposes here. In order to assess the quality of the direct simulations a detailed grid refinement study was conducted. We used grids with $64^{3}, 96^{3}, 128^{3}, 192^{3}, 256^{3}$, and $384^{3}$ grid cells and considered the convergence of the predictions on these grids.

For the grid convergence we first turn to the flow at initial $\operatorname{Re}_{\lambda}=50$. In Fig. 1(a) the total energy decay at different resolutions is plotted. Similarly, the Taylor length scale at different resolutions is displayed in Fig. 1(b). It is clear that the convergence is quite good, especially for the energy in which case all included resolutions appear to yield virtually the same result. In contrast, the Taylor length scale displays a more gradual convergence and it appears that the $192^{3}$ and the $256^{3}$ grids provide accurate predictions.

Apart from such visual inspection, convergence may be further checked in a more rigorous way. This also allows one to estimate the remaining error in the DNS. Consider solution properties $\phi^{n}$ obtained on three different grids with $n_{1}^{3}$, 

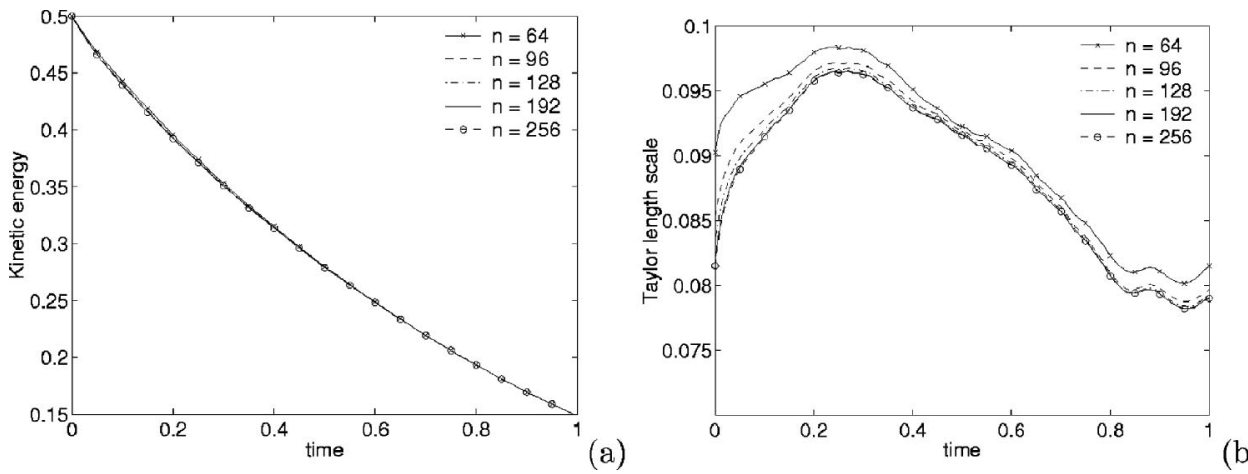

FIG. 1. Direct simulation results for the case $\mathrm{Re}_{\lambda}=50$ : (a) total energy decay at different resolutions; (b) Taylor length scale evolution at different resolutions.

$n_{2}^{3}$, and $n_{3}^{3}$ grid cells and corresponding mesh sizes $1 / n_{1}$, $1 / n_{2}$, and $1 / n_{3}$, assuming $n_{1}<n_{2}<n_{3}$. If the mesh sizes are sufficiently small a $p$ th order accurate numerical method implies

$$
\begin{aligned}
& \frac{\phi^{n_{3}}-\phi^{n_{2}}}{\phi^{n_{2}}-\phi^{n_{1}}}=\frac{1-\left(n_{2} / n_{3}\right)^{p}}{\left(n_{2} / n_{1}\right)^{p}-1}, \\
& \phi^{\mathrm{ex}}-\phi^{n_{3}}=\epsilon^{n_{3}}=\left(\phi^{\left.n_{3}-\phi^{n_{2}}\right)} \frac{\left(n_{2} / n_{3}\right)^{p}}{1-\left(n_{2} / n_{3}\right)^{p}} .\right.
\end{aligned}
$$

At sufficient resolution the order of the discretization method can be determined from Eq. (9). Moreover, Eq. (10) allows one to estimate the error on the $n_{3}$ grid as the difference of the exact solution $\phi^{\text {ex }}$ with $\phi^{n_{3}}$.

In principle, Eqs. (9) and (10) are valid only in case the resolution is already quite high. This condition needs to be checked independently, e.g., by repeating the determination of the order of the method for several grid sequences. We illustrate this for the predictions of the Taylor length scale. In Fig. 2(a), the estimated order $p$ during the calculation is plotted for two combinations of grid refinements, i.e., 96-128 -192 and 128-192-256. It is clear that the predictions of $p$ for both refinement series are basically the same as a function of time. Furthermore, $p$ is seen to vary between 2 and 4 , which corresponds with the formal order of the spatial discretization schemes used for the convective and viscous fluxes, i.e., fourth and second order, respectively. In Fig. 2(b) the resulting absolute error estimate is plotted for both grid- refinement series. From this it is clear that the simulation error on the $256^{3}$ grid is lower than $0.5 \times 10^{-3}$ in absolute value. This corresponds to a relative error for the Taylor length scale lower than $0.7 \%$. Similarly, the relative error for the total energy has an upper limit of $0.06 \%$, while the relative error for the skewness of the longitudinal velocity derivative $S_{3}$ does not exceed $6 \%$.

For the case in which the initial $\operatorname{Re}_{\lambda}=100$, a similar analysis was performed. Here, the spatial discretization scheme is fourth-order accurate for the convective as well as for the viscous terms. The major incentive to switch to a fourth-order scheme for the viscous terms was the observation that in case $\operatorname{Re}_{\lambda}=50$ a fully fourth-order scheme converges already on $128^{3}$ grid cells to within the relative error levels established earlier. In case $\operatorname{Re}_{\lambda}=100$ the convergence analysis leads to the requirement to use resolutions of up to $384^{3}$ grid points for the fully fourth-order scheme. Without treating the viscous terms with the fourth-order scheme an estimated resolution of $768^{3}$ grid points would have been required. The two grid sequences used for the $\operatorname{Re}_{\lambda}=100$ convergence study were $128-192-256$ and $192-256-384$. A similar analysis as before established that the finest grid indeed is adequate with relative error levels for the total kinetic energy and Taylor length scale estimated as $0.06 \%$ and $3 \%$. For the skewness $S_{3}$, the predictions for $p$ do not coincide. A crude error estimation indicates that $S_{3}$ (in absolute value) is approximately $20 \%$ too low.
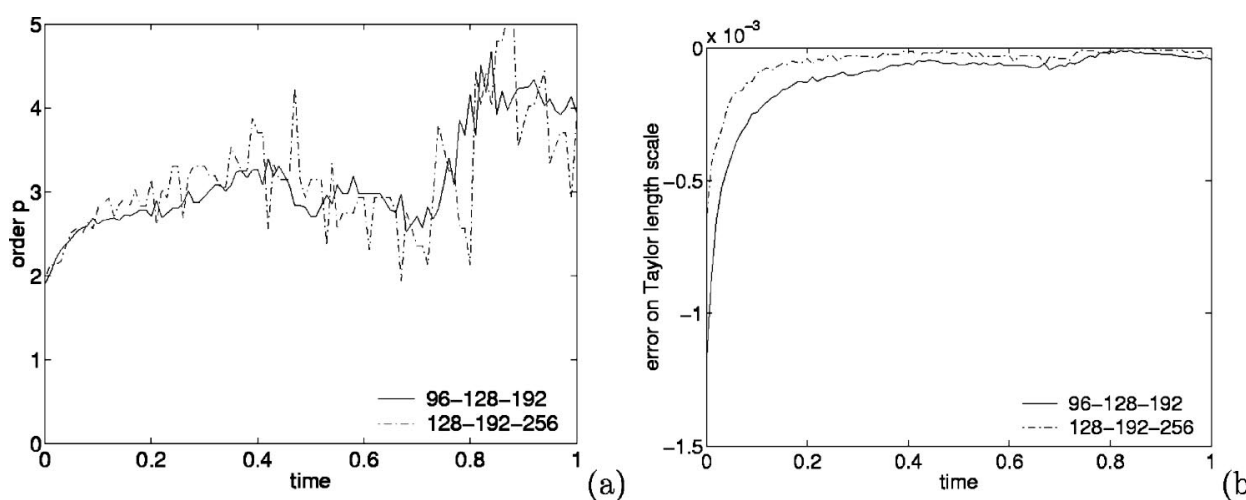

FIG. 2. Direct simulation results for the case $\operatorname{Re}_{\lambda}=50$ : (a) order $p$ for two different grid-refinement sets calculated by using the convergence of the Taylor length scale; (b) estimated error on the Taylor length scale for the $192^{3}$ and $256^{3}$ grids. 

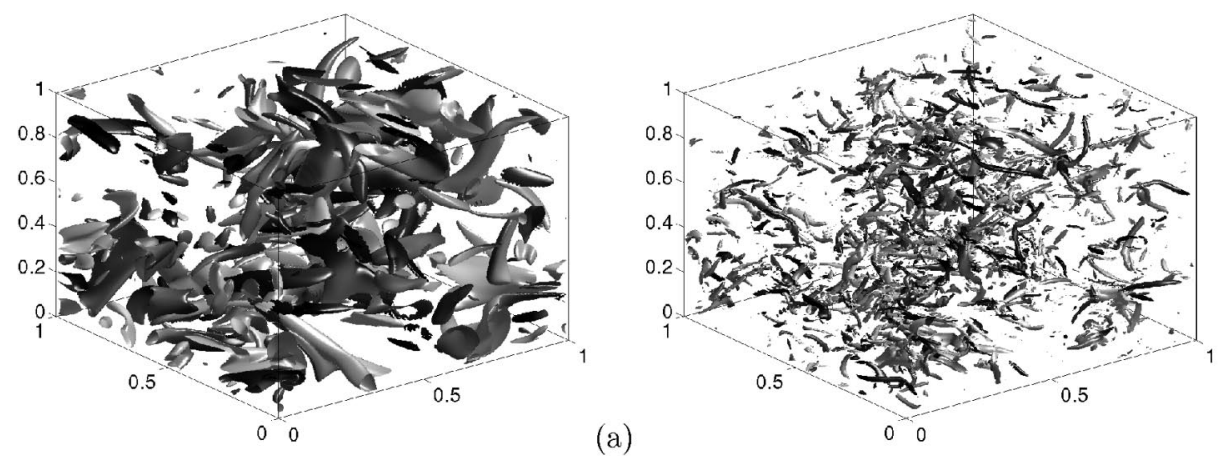

(b)

FIG. 3. Snapshots of vorticity: (a) at $t=0.6$ and $\operatorname{Re}_{\lambda}=50$, (b) at $t=0.6$ and $\operatorname{Re}_{\lambda}=100$. Isolevels for (a) and (b) at $0.25|\omega|_{\max }$.

\section{B. Direct numerical simulation results}

In this section we present some direct numerical simulation results obtained on the finest grids, i.e., $256^{3}$ as $\operatorname{Re}_{\lambda}$ $=50$ and $384^{3}$ as $\operatorname{Re}_{\lambda}=100$. In Fig. 3 a typical snapshot of the vortical structures in the simulated homogeneous isotropic turbulence is presented. With increasing Reynolds number a corresponding strong increase in the amount of smallscale structures can readily be appreciated. The vortical structures in Fig. 3 were visualized by directly plotting isosurfaces of the magnitude of the vorticity, i.e., $|\omega|$ $=\left(\omega_{i} \omega_{i}\right)^{1 / 2}$ where $\omega_{i}$ is the $i$ th component of $\boldsymbol{\nabla} \times \mathbf{u}$. These snapshots provide only a first global impression of the flow. We proceed by considering the Reynolds number dependence of some central properties.

In Fig. 4(a) the total kinetic energy decay for both Reynolds numbers is plotted. The energy decay is virtually identical in both cases. This corresponds to the fact that at high
Reynolds numbers, the inertial-range turbulent scales of any flow display to some degree universal behavior. Consequently, also the large scale properties become Reynolds independent. The turbulent kinetic energy is such a large scale property and is mainly determined by the "energy containing eddies." The small differences between the energy decay for both Reynolds numbers can be understood by recognizing that $\operatorname{Re}_{\lambda}=50$ is rather low for the existence of a universal turbulent equilibrium range.

The evolution of the Taylor length scale is presented in Fig. 4(b) while the corresponding Taylor Reynolds number is plotted in Fig. 4(c). In these plots, a transition interval between $t=0.0$ and $t=0.2-0.4$ is clearly discernible. In this time frame the Taylor Reynolds number grows to a maximum value before it starts to decrease monotonously. This is not related to actual physical behavior of decaying homogeneous isotropic turbulence, but instead results mainly from
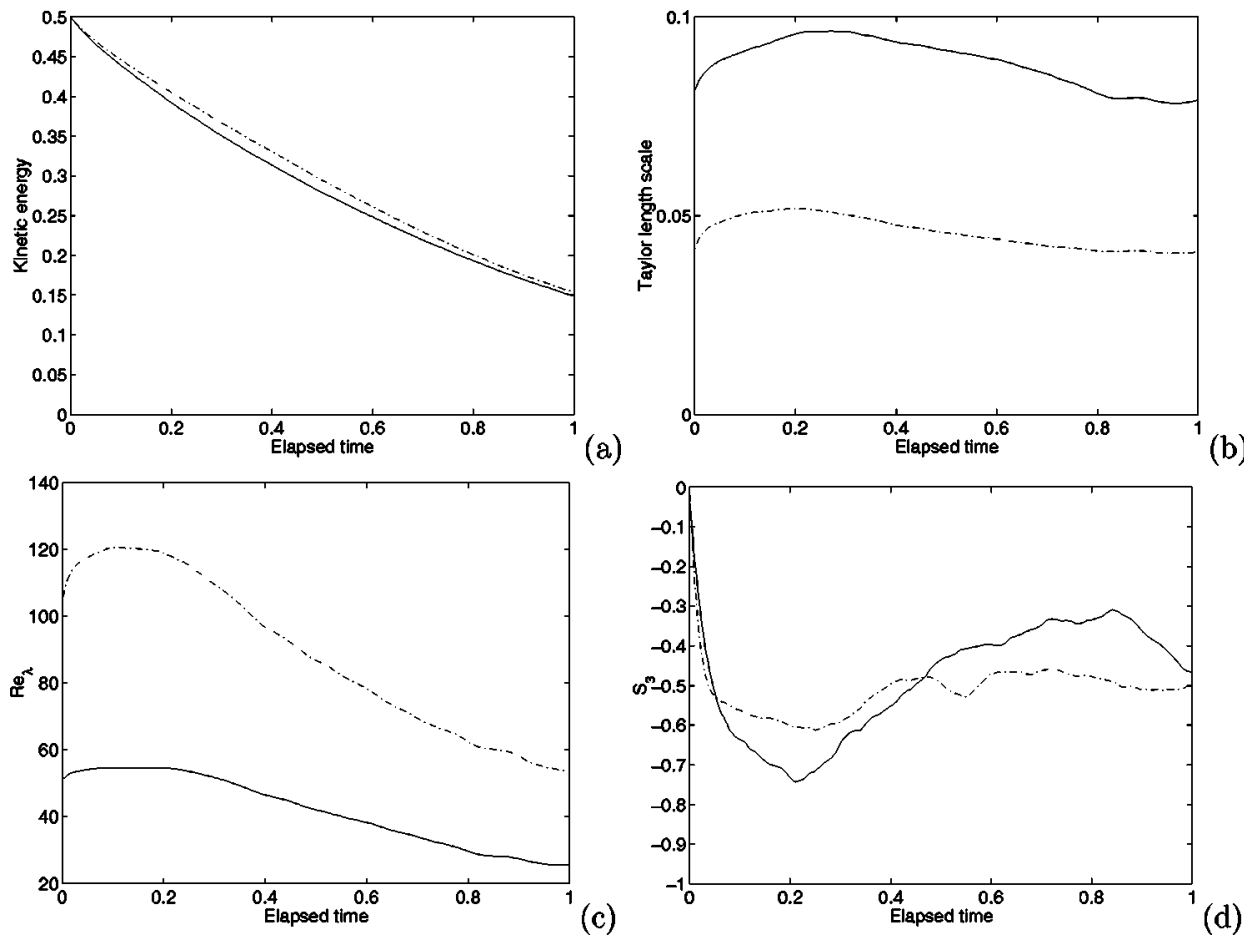

FIG. 4. (a) Energy decay for DNS of homogeneous isotropic turbulence; (b)Taylor length scale; (c) Taylor Reynolds number; (d) skewness of the longitudinal velocity derivative. Solid line: $\mathrm{Re}_{\lambda}=50$; dash-dotted line: $\mathrm{Re}_{\lambda}=100$ for all plots. 
the evolution of the initial velocity field toward a physically realistic turbulent velocity field. Primarily, in this time frame phase correlations are developing properly among the Fourier modes. Indeed, although the initial velocity field satisfies the continuity equation and corresponds to a relevant energy spectrum, the phases of the initial velocity are random and the initial and short-time fields do not correspond to a physical flow.

In Fig. 4(d), the skewness of the longitudinal velocity derivative is shown. Again, the transition region is clearly visible in which the skewness evolves from 0 to a value of about -0.5 . This is in good agreement with other data on the skewness of the longitudinal velocity derivative, which range for moderate to high Reynolds numbers from ${ }^{36}-0.3$ to -0.9 . After the transition region, the skewness is nearly constant in time in case $\operatorname{Re}_{\lambda}=100$. This indicates the existence of a universal equilibrium range. ${ }^{37}$ For $\operatorname{Re}_{\lambda}=50$ the skewness varies considerably more, which indicates that this Reynolds number is too low to display a universal equilibrium range.

\section{The LES approach: Fundamentals and simulation setup}

In this section we formulate the incompressible LES approach that is followed here and describe the setup of the individual large-eddy simulations.

The filtered, incompressible Navier-Stokes equations can be written as

$$
\partial_{t} \bar{u}_{i}+\partial_{j}\left(\bar{u}_{i} \bar{u}_{j}\right)+\partial_{i} \bar{p}-\frac{2}{\operatorname{Re}} \partial_{j} \bar{S}_{i j}=\partial_{j} \tau_{i j}, \quad i=1,2,3,
$$

where the spatial convolution filter is denoted by $\overline{(\cdot)}$ and the filtered solution is given by $\left\{\bar{u}_{i}, \bar{p}\right\}$. The filtering of the convective terms gives rise to the divergence of the turbulent stress tensor

$$
\tau_{i j}=\bar{u}_{i} \bar{u}_{j}-\overline{u_{i} u_{j}} .
$$

In addition,

$$
\bar{S}_{i j}=\frac{1}{2}\left(\frac{\partial \bar{u}_{i}}{\partial x_{j}}+\frac{\partial \bar{u}_{j}}{\partial x_{i}}\right)
$$

denotes the filtered rate of strain tensor. Since the left-hand side of Eq. (11) corresponds to the Navier-Stokes operator NS acting on the filtered solution $\left\{\bar{u}_{i}, \bar{p}\right\}$ (instead of $\left\{u_{i}, p\right\}$ ), the large-eddy approach can be expressed in short-hand notation as

$$
\mathrm{NS}\left(\bar{u}_{i}\right)=\frac{\partial \tau_{i j}}{\partial x_{i}} .
$$

Hence, the filtered solution obeys Navier-Stokes dynamics in which the subgrid-scale stress term is responsible for the smoothing of the turbulent flow and pressure fields. Although the filter determines all aspects of the closure problem for $\tau_{i j}$, in virtually all actual large-eddy simulations the filter operator has only formal significance and is not performed explicitly in a simulation. ${ }^{24}$ Ideally, the filter information should be retained in explicit models of the turbulent stress tensor. In practice, this is not the case and most existing subgrid models only indirectly contain features of the adopted filter such as the filter width $\Delta$.

In this paper we adopt the Smagorinsky subgrid model $m_{i j}^{S}$ which implies

$$
\tau_{i j} \rightarrow m_{i j}^{S}=2\left(C_{\mathrm{S}} \Delta\right)^{2}\left[\sqrt{\left\langle 2 \bar{S}_{i j} \bar{S}_{i j}\right\rangle}\right] \bar{S}_{i j},
$$

where $\langle\cdot\rangle$ denotes a volume average. In this model the smoothing of the solution is governed by the product of the Smagorinsky constant $C_{\mathrm{S}}$ and the filter-width $\Delta$. The value of the Smagorinsky constant can be estimated provided some additional assumptions about the nature of the turbulent flow are invoked. In literature various values have been suggested, mainly ranging from $C_{\mathrm{S}}=0.1$ to $C_{\mathrm{S}}=0.2$, e.g., Refs. $38-40$. However, we will not decide on a specific value for $C_{\mathrm{S}}$ here, but rather consider the product $C_{\mathrm{S}} \Delta$ as the relevant parameter which we will refer to as the effective filter width. An increase in the effective filter width will give rise to a decrease in the wavenumber content of the numerical solutions while the full turbulent flow is obtained in case the effective filter width is reduced to zero.

Next to the effective filter width the grid spacing $h$ of the computational grid is an important parameter in the definition of the large-eddy approach. At constant effective filter width a decrease in $h$ will reduce the importance of discretization errors and gradually give rise to a "grid-independent" large-eddy solution corresponding to the Smagorinsky model. The ratio $\Delta / h$ will be referred to as the subgrid resolution. To achieve a good approximation of the gridindependent solution a subgrid resolution of up to 6-8 may be required. ${ }^{31}$ However, in most practical LES, the computational grid is not only considerably coarser than the corresponding DNS grid, but-to reduce costs-also much coarser than the dynamics of the LES equations would require for a "grid independent" solution. Typically, subgrid resolutions of about 1-2 are adopted in applied flow research. In the latter case the influence of spatial discretization effects may be considerable. By systematically varying the subgrid resolution one may infer the contaminating discretization effects from the database approach as we will show momentarily.

For the Smagorinsky model, and similarly for other subgrid scale models, the (effective) filter width reduces the dynamic contents of the filtered Navier-Stokes equations [Eq. (14)] and only large turbulent scales remain in the LES solution. Hence $\Delta$ induces a truncation of the Navier-Stokes dynamics. This article focuses on the use of finite difference based discretization techniques. The emphasis is on determining the effects of numerics within the already truncated dynamics, for a standard LES system comprised of a basic spatial discretization and the Smagorinsky model.

The initialization of the individual large-eddy simulations is similar to the DNS initialization outlined before. The same prescribed energy spectrum is imposed and the same random number series is used for the initialization of the phases in order to be able to compare the solutions obtained at different resolutions. In fact, consider two resolutions characterized by $n_{1}$ and $n_{2}$ with $n_{1}<n_{2}$. We generate the 
Fourier components of the initial solution on the $n_{2}$ grid such that it contains exactly the same field as the $n_{1}$ grid for the modes shared by both grids. To these modes the required number of additional small scale components are added to complete the initial solution on the $n_{2}$ grid. Roughly speaking, the initial fields are generated "from inside out" in terms of the wavenumber $k$. Filtering aspects of the generation of the initial data will be further discussed in Sec. III B. Further, the subgrid fluxes are determined using the same secondorder spatial discretization method as used for the viscous fluxes. Corresponding to one direct numerical simulation at a given $\operatorname{Re}_{\lambda}$ a large number of associated large-eddy simulations is obtained by variation of the effective filter width and the numerical resolution. In this way the modeling and numerical error contributions to the total error can be individually controlled, which is the central point of departure for the database analysis.

In Sec. III we will introduce specific measures for the simulation errors and consider a detailed error decomposition which allows one to assess the magnitude of the individual errors.

\section{QUANTIFICATION OF INTERACTING ERROR DYNAMICS}

In Sec. III A, a detailed error decomposition is presented for the decay of the kinetic energy. The methodology used was first presented in Vreman et al. ${ }^{29}$ and illustrated for turbulent mixing layers. Here, we apply the analysis to homogeneous turbulence and consider the effects of Reynolds number and numerical parameters on the error interactions. Subsequently, in Sec. III B the role of the LES filter in the definition of errors is studied. This leads to two related but different definitions of the error contributions in LES, in which LES is either compared with filtered or unfiltered DNS data. We will establish the robustness of the primary error behavior in terms of these error measures.

\section{A. Detailed error decomposition for LES}

It is common practice to compare LES predictions with filtered DNS data. For this purpose we introduce an explicit filter operation for which we adopt a Gaussian filter. In one spatial dimension this can be written as a convolution

$$
\bar{\phi}(x, t)=\int_{-\infty}^{\infty} G(x-\xi) \phi(\xi, t) d \xi
$$

with $G(x-\xi)$ the filter kernel which is given by

$$
G(x-\xi)=\left(\frac{6}{\pi \Delta^{2}}\right)^{1 / 2} \exp \left(-\frac{6(x-\xi)^{2}}{\Delta^{2}}\right)
$$

In three spatial dimensions filtering implies the consecutive application of three one-dimensional filters. The filtering operation is performed by Fourier transforming the DNS solution field, applying spatial convolution as a product in Fourier space, and inverting the Fourier transform. Instead of the Gaussian filter one could also consider the top-hat filter and obtain both qualitatively and quantitatively similar results. ${ }^{41}$
In terms of the explicit filter it is now straightforward to define the different error contributions in LES. ${ }^{29}$ Consider a reference DNS and a variable of interest $\phi$, which may be any specific flow quantity. The total error in $\phi$, obtained from a LES, with filter width $\Delta$ and grid spacing $h$, is defined as

$$
e_{\text {total }}(\Delta, h)=\overline{\phi_{\mathrm{DNS}}}-\phi_{\mathrm{LES}}(\Delta, h) .
$$

Here, we explicitly identified the error as a function of the filter width and the grid spacing. This total error $e_{\text {total }}$ can be further decomposed into a contribution due to the discretization and the subgrid modeling, ${ }^{29}$ i.e.,

$$
e_{\text {total }}=e_{\text {model }}+e_{\text {discr }},
$$

where

$$
\begin{aligned}
& e_{\text {model }}=\overline{\phi_{\mathrm{DNS}}}-\phi_{\mathrm{LES}}(\Delta, 0), \\
& e_{\mathrm{discr}}=\phi_{\mathrm{LES}}(\Delta, 0)-\phi_{\mathrm{LES}}(\Delta, h) .
\end{aligned}
$$

In these expressions $\phi_{\mathrm{LES}}(\Delta, 0)$ represents the gridindependent prediction obtained from the Smagorinsky modeled LES equations with filter-width $\Delta$. This "gridindependent LES" prediction provides a central point of reference in the error decomposition, next to the reference DNS data.

"Grid-independent" LES does not correspond to "practical" large-eddy simulation as practitioners in the field would typically consider. Roughly speaking, it is a LES on a DNS grid in which the filter-width $\Delta$ is much larger than the mesh-spacing. Consequently, the "grid-independent" LES also contains contributions to length scales in the solution which are much smaller than the filter width. However, the dynamic importance of these "sub- $\Delta$ " flow components in the modeled solution is very small due to the action of the subgrid fluxes, e.g., through extra dissipation associated with the Smagorinsky model.

The high-resolution predictions at fixed filter width provide a point of reference in which discretization error effects are eliminated as much as possible from the solution to the modeled LES equations. After all, in one interpretation of large-eddy simulation, the continuous, modeled LES equations constitute a closed system of nonlinear partial differential equations with fixed, externally specified length-scale given by the filter-width $\Delta$. The continuous solution to these equations is approximated accurately with the "gridindependent" LES. Note that this yields, e.g., the gridindependent solution to what could be called a "Smagorinsky fluid," which is not necessarily also an accurate approximation to filtered DNS predictions. All that is minimized in such "grid-independent" LES is the discretization error in the solution to the modeled equations corresponding to a particular subgrid model. Clearly, grid-independent LES requires very high resolutions and is not feasible (nor required) in many cases. As such, "grid-independent" LES should not be confused with a proposal to perform actual, practical LES in this way.

The "grid-independent" LES allows for a detailed interpretation of the interacting error-dynamics and we will use it in this setting. It is introduced only to be able to assess the type and rate of convergence of numerical solutions toward 

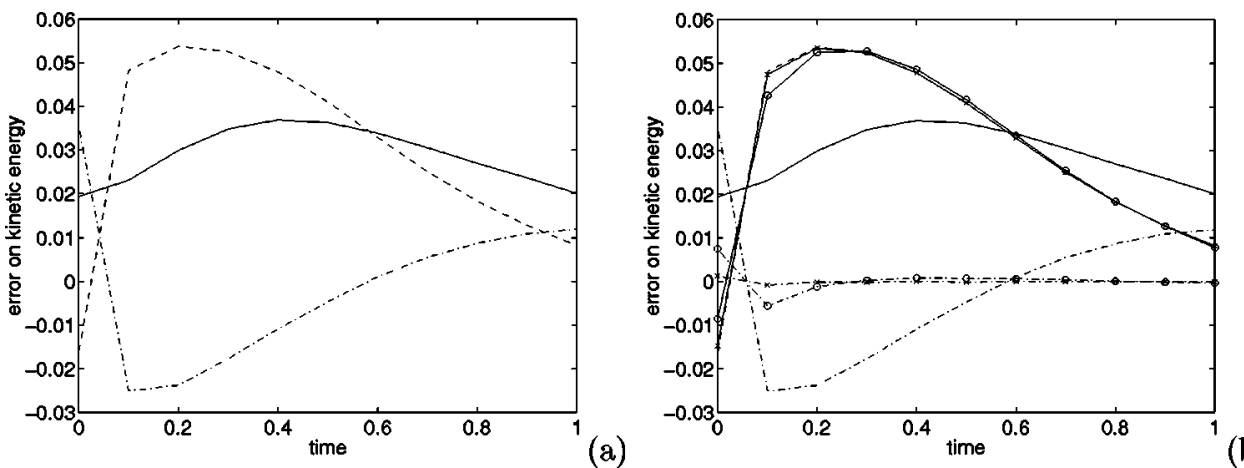

FIG. 5. (a) Error decomposition for a $32^{3}$ LES with $C_{\mathrm{S}} \Delta=h / 5=6.25 \times 10^{-3}$; (b) error decomposition of LES at $C_{\mathrm{S}} \Delta=6.25 \times 10^{-3}$ for three different resolutions: (no symbol) $32^{3} ;(\bigcirc) 64^{3} ;(\times) 96^{3}$. In both figures: (-) total error; (---) modeling error; (-.-) discretization error.

the continuous solution of the modeled LES equations, i.e., in this paper the system of flow equations including the Smagorinsky closure. The grid-independent solution does not correspond to the most accurate LES in the sense of minimal total error. In this section we illustrate the reasons for this in some detail.

We do not consider errors due to the temporal integration. We adopted an explicit Runge-Kutta time-stepping method and restricted the time step in order to comply with the stability requirements of this method. It was observed that the error associated with the time stepping is negligible compared to the numerical errors due to the spatial discretization.

The availability of reference DNS data as well as approximately grid-independent LES predictions allows one to fully separate the contributions to the total error. We concentrate on the decay of the kinetic energy in order to illustrate the approach. In Fig. 5(a), the error decomposition for LES on a grid with $32^{3}$ grid cells, i.e., $h=1 / 32$, is compiled. We adopted an effective filter-width $C_{\mathrm{S}} \Delta=h / 5=6.25 \times 10^{-3}$ and $\operatorname{Re}_{\lambda}=100$. At a typical value for the Smagorinsky constant of $C_{\mathrm{S}}=0.2$, this setting corresponds to the case in which the filter width equals the grid spacing on the $32^{3}$ grid, i.e., $\Delta / h=1$. The grid-independent LES, keeping $C_{\mathrm{S}} \Delta$ constant, is approximated at a resolution of $128^{3}$. We verified independently that the grid independence of this LES is satisfactory; in fact, a resolution of $96^{3}$ would already have been quite adequate for the error decomposition. It is clearly seen that for times up to $t \approx 0.6$ the total error is lower in absolute value than one of its contributing components. This is due to the fact that the numerical and discretization errors counteract in this time frame. This gives rise to a counter-intuitive situation in which an improved simulation, e.g., at higher spatial resolution, will yield results with a larger total error. In fact, the proposed "improved simulation" corresponds to a LES refinement toward the grid independent solution associated with the adopted subgrid model. In the present case such increased resolution simulations not only significantly increase the computational cost but these simulations may also be suboptimal with respect to accuracy in terms of differences compared to filtered DNS predictions. As an example, grid refinement at constant $C_{\mathrm{S}} \Delta$ results in a deterioration of the LES results as can be observed in Fig. 5(b). In this figure results on $32^{3}$ grid cells are compared with predictions at $64^{3}$ and $96^{3}$ cells, all with $C_{\mathrm{S}} \Delta=6.25 \times 10^{-3}$. We observe that the discretization error effect decreases corresponding to the improved numerical capturing of the Smagorinsky solution. Rather than giving rise to a smaller total error we notice that the total error approaches the modeling error which by itself is larger than the total error on coarser grids. Similar counteracting error behavior was also observed in turbulent mixing layers. ${ }^{29}$

In Fig. 6(a) modeling errors are plotted for a $32^{3}$ LES at different filter widths. The magnitude of the Smagorinsky term decreases with decreasing filter width. Figure 6(b) displays the corresponding discretization error effects. Here, the reverse tendency may be observed, i.e., the discretization error effects increase (in absolute value) with decreasing filter width. In fact, due to a decreased filter width, the LES solution becomes less smooth, since the effective viscosity is decreased, and therefore the numerical LES solution is represented less accurately on the same grid. As before, we observe that both errors have opposite signs. An overview of modeling, discretization, and total error effects is collected in Fig. 6(c). We notice that at $C_{\mathrm{S}} \Delta=0.2 h$ the total error is smaller than in the case $C_{\mathrm{S}} \Delta=0.1 \mathrm{~h}$ or $C_{\mathrm{S}} \Delta=0.3 h$. This arises from the different strengths of the counteracting modeling and discretization errors for the three different largeeddy simulations. These appear balanced best at $C_{\mathrm{S}} \Delta$ $=0.2 \mathrm{~h}$. At $C_{\mathrm{S}} \Delta=0.1 \mathrm{~h}$, the discretization error clearly dominates the total error, while at $C_{\mathrm{S}} \Delta=0.3 h$ the modeling error is dominant.

To illustrate the error contributions for flow properties that involve a stronger contribution of the smaller scales we consider the Taylor length scale in Fig. 7. The error decomposition for a $32^{3}$ LES with different filter widths shows that the discretization and modeling error effects do not counteract. Again, we observe a decreasing modeling error effect with decreasing filter width and a corresponding increase in the discretization error effect. The decrease in the modeling error effect with decreasing $C_{\mathrm{S}} \Delta$ is much stronger than the increase in the discretization error effect for these three cases. Since the error effects have the same sign, the Taylor length scale is predicted most accurately in case the effective filter width is smallest, i.e., for these three cases at $C_{\mathrm{S}} \Delta$ $=0.1 h$. 

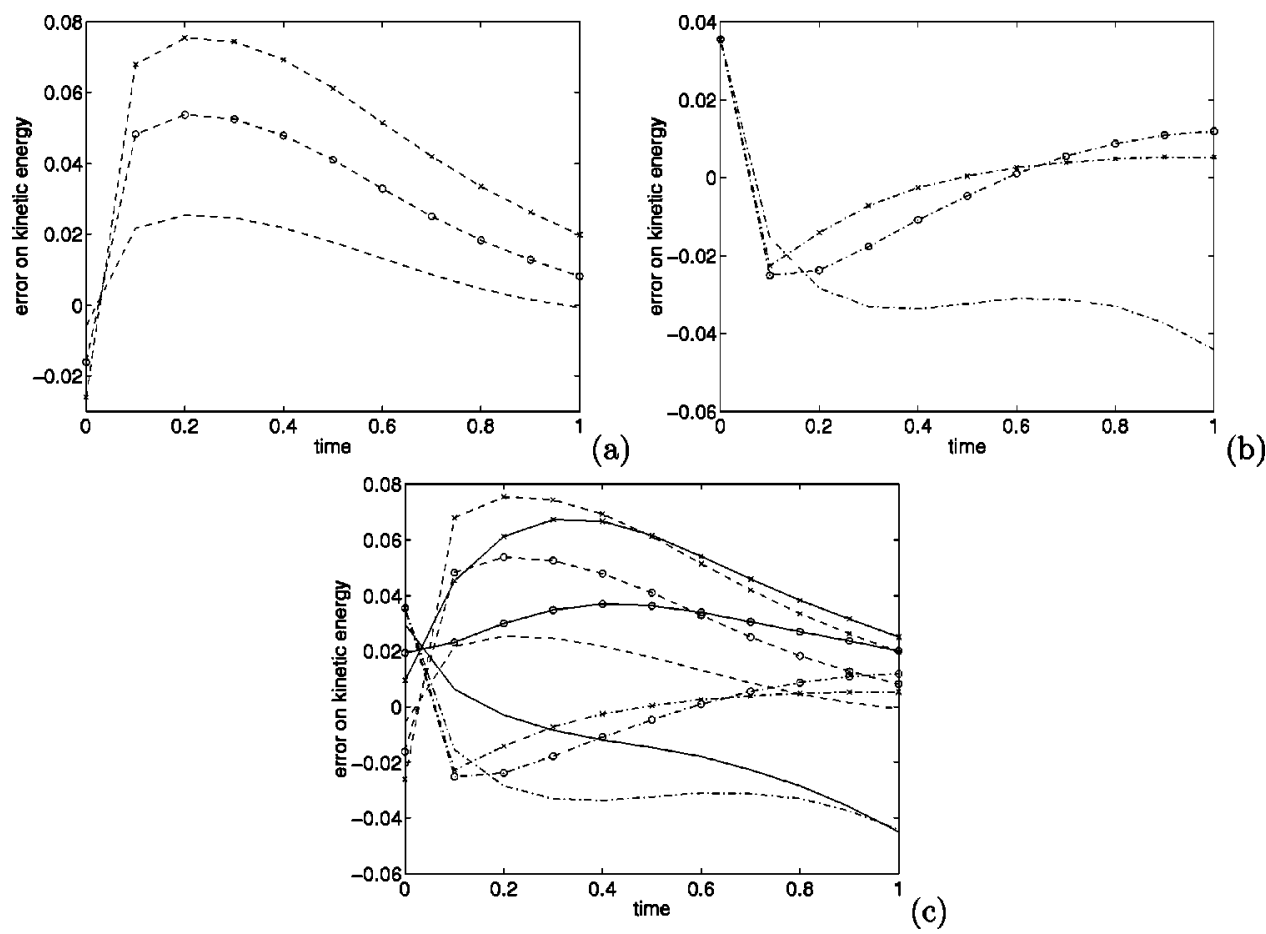

FIG. 6. Modeling error (a) and numerical error (b) for a $32^{3}$ LES at different filter widths: (no symbol) $C_{\mathrm{S}} \Delta=0.1 h ;(\bigcirc) C_{\mathrm{S}} \Delta=0.2 h ;(\times) C_{\mathrm{S}} \Delta=0.3 h$. In (c) the total error (-), the modeling error (---) and the discretization error (-.-) are collected.

The presented error decomposition provides a detailed view of the intricate error interactions in LES. For certain numerical and physical modeling parameters the basic errors may counteract. This leads to an intriguing paradox related to possible strategies that should be followed to further improve LES predictions compared to some reference simulation. While it is tempting to think that a higher resolution, a better numerical method, or a more precise subgrid model would always lead to improved accuracy of the predictions, the counteracting property of the errors and their specific reverse dependence on filter width can completely distort this impression. Rather, the total error arises from a balance between modeling and discretization errors. Thus, it is not an easy matter to predict a priori whether these errors will or

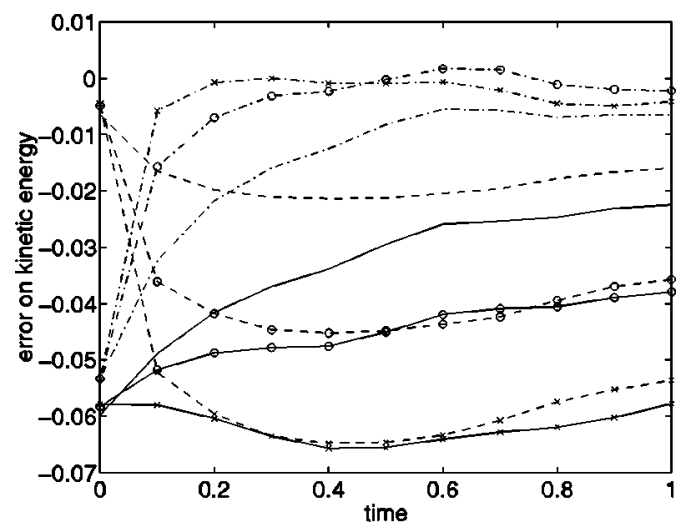

FIG. 7. Error contributions on Taylor length scale in a $32^{3}$ LES at different filter widths: (no symbol) $C_{\mathrm{S}} \Delta=0.1 h ;(\bigcirc) C_{\mathrm{S}} \Delta=0.2 h ;(\times) C_{\mathrm{S}} \Delta=0.3 h$. The total error (-), the modeling error (---), and the discretization error $(-\cdot)$ are collected. will not counteract and what the magnitude of the individual error contributions is. In particular, these error contributions and the type of interactions will depend also on the numerical and physical parameter setting. An overview of this behavior can be obtained from a full database analysis of a systematically varied set of large-eddy simulations which we will elaborate in Secs. IV and V. Before turning to this analysis we first consider the role of the explicit filter in the definition of the error measures and the robustness of the corresponding error behavior in Sec. III B.

\section{B. Errors and filtering}

The point of reference for the error analysis in Sec. III A

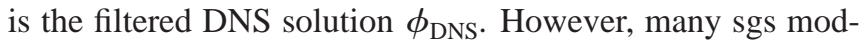
els do not explicitly determine the LES filter, although in view of Eq. (14), the sgs model is responsible for the smoothing of the LES solution. In particular, the filter is not explicitly defined for the Smagorinsky model considered here. The only input for the model is the product $C_{\mathrm{S}} \Delta$. Hence, not only the filter shape but also the filter width are not strictly defined. In literature, different values for $C_{\mathrm{S}}$ are quoted, ranging from $0.1,^{38} 0.15,{ }^{35} 0.17,{ }^{39}$ to $0.21 .^{40}$ These values are determined, e.g., through a determination of the "implicit" Smagorinsky filter shape ${ }^{35,40,42}$ or through empirical fitting of the model to DNS results, for example. ${ }^{38,39}$ Once a value for $C_{\mathrm{S}}$ is fixed, the filter width also attains a definite value. Figure 8 displays the total errors in the kinetic energy arising in one and the same $32^{3}$ LES at $C_{S} \Delta=0.2 h$ as a function of $C_{\mathrm{S}}$. The different filters correspond, respectively, to $C_{\mathrm{S}}=0.1,0.15,0.2$, and $\infty$ (the last one indicating no filtering at all) and each provides a different $\overline{\phi_{\text {DNS }}}$. The 


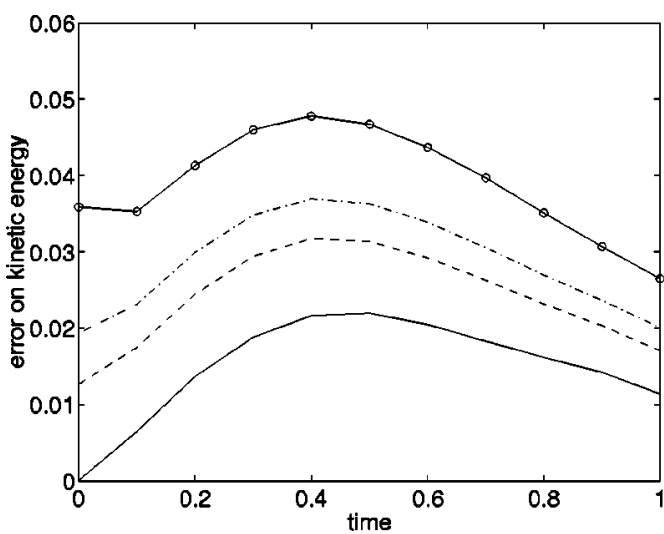

FIG. 8. Influence of the filter uncertainty on the total kinetic error level for a $32^{3}$ LES at $C_{\mathrm{S}} \Delta=6.25 \times 10^{-3}$ and $\operatorname{Re}_{\lambda}=100$. Filter corresponding to $C_{\mathrm{S}}$ $=0.1(-), C_{\mathrm{S}}=0.15(---) ; C_{\mathrm{S}}=0.2(-\cdot-)$; no filter (-O-).

differences in error levels arising from the filter uncertainty appear quite relevant although the global error evolution is of comparable nature. In fact, the total error lines are related to one another roughly by a relative shift corresponding to the total error level at $t=0$.

Formally, it is possible to define a priori the shape and width (corresponding to a value of $C_{\mathrm{S}}$ ) of the LES filter and also apply this filter explicitly on the initial truncated LES field. For all LES presented in the current paper, only truncated initial conditions are considered and no extra filter is added on these fields. The influence of an explicit initial filter was checked for Gaussian filters corresponding to $C_{\mathrm{S}}=0.1$, $0.15,0.2$ and various settings of $C_{\mathrm{S}} \Delta$. The difference in total error as a result of this initial filter operation is found to be an order of magnitude lower than the level of the respective errors themselves and negligible in most regions.

From the analysis shown in Fig. 8, it is clear that the uncertainty in the filter width and-presumably to a lesser extent - the filter shape has to be contributed for in a LES error analysis. In order to avoid this uncertainty in the filterwidth definition, an alternative definition of the error measures is considered next to the classical definition given in Ref. 29. For this purpose and to investigate the robustness of the error behavior and corresponding conclusions we consider an alternative error $\hat{e}$ based on a comparison with the unfiltered DNS predictions $\phi_{\text {DNS }}$ instead of $\overline{\phi_{\text {DNS }}}$. Hence, we distinguish

$$
\begin{aligned}
& \hat{e}_{\text {total }}=\phi_{\mathrm{DNS}}-\phi_{\mathrm{LES}}(\Delta, h), \\
& \hat{e}_{\mathrm{discr}}=\phi_{\mathrm{LES}}(\Delta, 0)-\phi_{\mathrm{LES}}(\Delta, h)=e_{\mathrm{discr}}, \\
& \hat{e}_{\text {model }}=\phi_{\mathrm{DNS}}-\phi_{\mathrm{LES}}(\Delta, 0)=e_{\text {model }}+\left(\phi_{\mathrm{DNS}}-\overline{\phi_{\mathrm{DNS}}}\right) .
\end{aligned}
$$

We observe that this change in definition does not affect the discretization error. With this error definition the modeling error is not zero even in case a "perfect" subgrid model would have been adopted. In fact, only $e_{\text {model }}$ would be zero in this case and $\hat{e}_{\text {model }}$ also includes the difference between filtered and unfiltered DNS data. The main benefit of this alternative error decomposition is that no explicit filtering is required and the above-mentioned filter uncertainties are hence avoided.

In the remainder of this paper, both error definitions will be used and the corresponding error behavior will be compared. Clearly, the error levels can differ considerably. However, as will be demonstrated, the primary findings related to optimal simulation strategies are quite independent of these definitions. In Sec. IV we introduce the "subgrid activity" parameter and consider the global error behavior. Moreover, we identify parameter settings in which either the modeling or the discretization error is dominant.

\section{SUBGRID ACTIVITY AND GLOBAL ERROR BEHAVIOR}

The global error behavior is studied here as function of relevant numerical and physical parameters. An extensive LES database was generated at different Reynolds numbers and a variety of resolutions and filter widths. We consider LES grids with $n^{3}$ grid cells where $n$ $\in\{16,24,32,48,64,96,128\}$ at $\operatorname{Re}_{\lambda}=50$. For $\operatorname{Re}_{\lambda}=100$ we also considered $n=80$. For sake of argument we assume $C_{\mathrm{S}}=0.2$ and consider a variety of filter widths $\Delta$ $\in\{1,2, \ldots, 9,10,12,14\} / 64$. We recall that the length of the side of the computational domain is set to one. The simulations at $n=128$ provide the approximately grid independent LES data. In total, several hundred separate large-eddy simulations were performed and in order to display the main trends in the error behavior the data are grouped in the same framework as proposed by Geurts and Fröhlich. ${ }^{31}$ Compared to this reference, the present database allows a much more detailed assessment of the error behavior. We consider the relation between total error and Reynolds number and the importance of the error definition. We include the kinetic energy, which is mainly dependent on large scale flow features, and the Taylor length scale, which is located in the inertial subrange and is an intermediate scale between the integral length scale and the Kolmogorov scale.

In order to measure the importance of the subgrid model in the evolution of the turbulent flow the subgrid activity parameter $s$ was introduced in Ref. 31. It is defined as

$$
s=\frac{\varepsilon_{t}}{\varepsilon_{t}+\varepsilon_{\nu}}
$$

with $\varepsilon_{t}$ the energy dissipation resulting from the sgs model and $\varepsilon_{\nu}$ the dissipation resulting from laminar viscosity, where $\varepsilon_{t}$ is calculated as $\left\langle m_{i j} \partial_{j} \bar{u}_{i}\right\rangle$ and $\varepsilon_{\nu}$ is obtained from $\left\langle 2 S_{i j} S_{i j}\right\rangle /$ Re. To obtain a single value $s$ for each LES run, the nearly constant subgrid activity Eq. (25) is averaged over time. Different averaging procedures were tested, but no significant influence on the resulting $s$ values were observed.

The subgrid activity $s$ quantifies the relative importance of the subgrid scale stresses. By definition it varies between 0 and 1 where a value of 0 corresponds to DNS and 1 is associated with LES at infinite Reynolds number. The value of $s$ is directly related to the filter-width $\Delta$ and measures the "distance" between a DNS resolving all flow features at sufficiently high spatial resolution and an actual LES corre- 

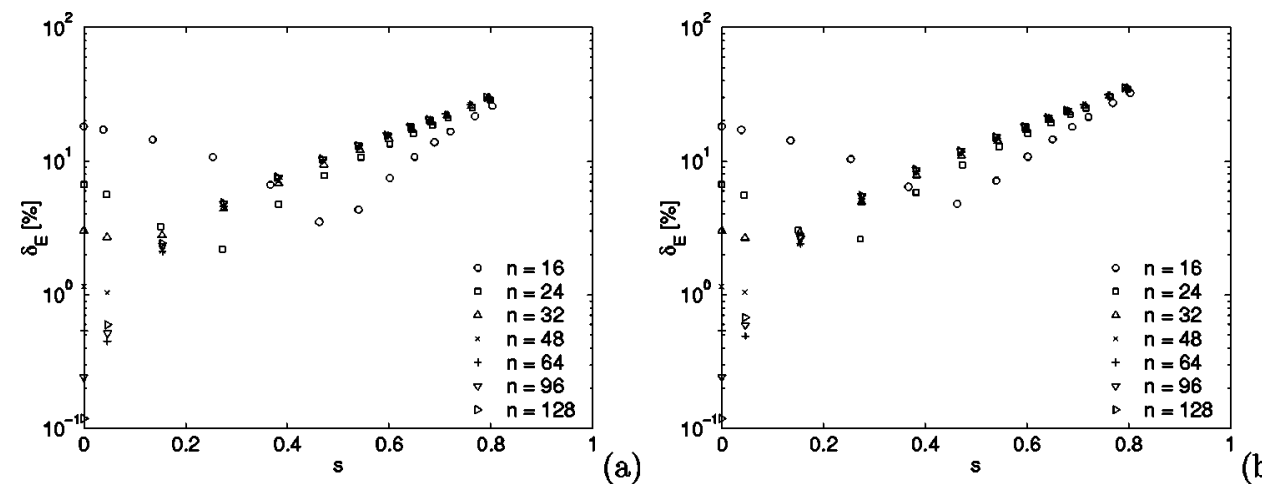

FIG. 9. Relative error $\delta_{E}$ (a) and $\hat{\delta}_{E}$ (b) for the kinetic energy at $\mathrm{Re}_{\lambda}=50$.

sponding to a specific filter width and mesh spacing. The estimate of $s$ for a particular LES was found to be very robust both at $\operatorname{Re}_{\lambda}=50$ and $\mathrm{Re}_{\lambda}=100$, similar to the findings reported in Ref. 31. The subgrid activity $s$ is preferred over the direct use of the filter-width $\Delta$ since the latter varies from flow to flow, depending on the nondimensionalization of the equations and, moreover, it is not well established for various popular subgrid models as was discussed in Sec. III B and in Ref. 24. In contrast, the definition of $s$ in Eq. (25) does not suffer from these shortcomings and can be applied for general subgrid models.

We concentrate on the total relative error which is defined as

$$
\delta=\frac{\int e_{\mathrm{total}}^{2} d t}{\int{\overline{\phi_{\mathrm{DNS}}}}^{2} d t}, \quad \hat{\delta}=\frac{\int \hat{e}_{\mathrm{total}}^{2} d t}{\int \phi_{\mathrm{DNS}}^{2} d t}
$$

in accordance with both error definitions in Sec. III. For the determination of $\overline{\phi_{\mathrm{DNS}}}$ a Gaussian filter is used. With this definition of the error measure each separate large-eddy simulation gives rise to a single real number. This global error definition makes it possible to effectively compare a large number of simulations in a single coherent framework.

In Fig. 9(a), the total error $\delta_{E}$ is plotted against the subgrid activity for the $\operatorname{Re}_{\lambda}=50$ case. We observe that for $s$ $\gtrsim 0.4$, the relative error as function of $s$ is quite independent of the resolution with the exception of the coarsest grids at $n=16,24$. In this regime the total error is dominated by modeling error effects. In fact, the large-eddy solutions are con- siderably smoothed and can already be represented accurately on coarse grids, i.e., $n \geqslant 32$. In this region $(s \gtrsim 0.4)$, the error is minimal at $s=0.4$ and grows exponentially with $s$ for $s \rightarrow 1$. For $s \lesssim 0.4$, the errors corresponding to different spatial resolutions are scattered considerably. In this region the total error is dominated by discretization error effects which are seen to introduce a considerable and clearly undesired amount of uncertainty in the actual LES predictions. The region around $s \approx 0.4$ defines the cross-over region between modeling or discretization dominated total error behavior. The value $s \approx 0.4$ roughly defines this cross-over point denoted by $s_{c}$. A similar error dependence was observed in Ref. 31 for LES of a temporal mixing layer. The cross-over point for this mixing layer flow was found at $s_{c}$ $\approx 0.5$ at a Reynolds number which is quite comparable to $\operatorname{Re}_{\lambda}=50$. Figure 9(b) represents the total error behavior evaluated with the alternative error definition $\hat{\delta}_{E}$. Although error levels differ slightly, the main pattern is clearly unchanged, including the cross-over point $s \approx 0.4$. Hence, the primary error pattern is robust with respect to the precise definition of the error measure.

In Fig. 10, the relative error behavior at $\operatorname{Re}_{\lambda}=100$ is shown. Again, both error measures give rise to the same error pattern, albeit at slightly different error levels. The main difference with the $\operatorname{Re}_{\lambda}=50$ case arises in a different cross-over point which is now located at $s_{c} \approx 0.8$.

In both Figs. 9 and 10, it is clear that for $s<s_{c}$, error levels can be lower or higher than the cross-over values,
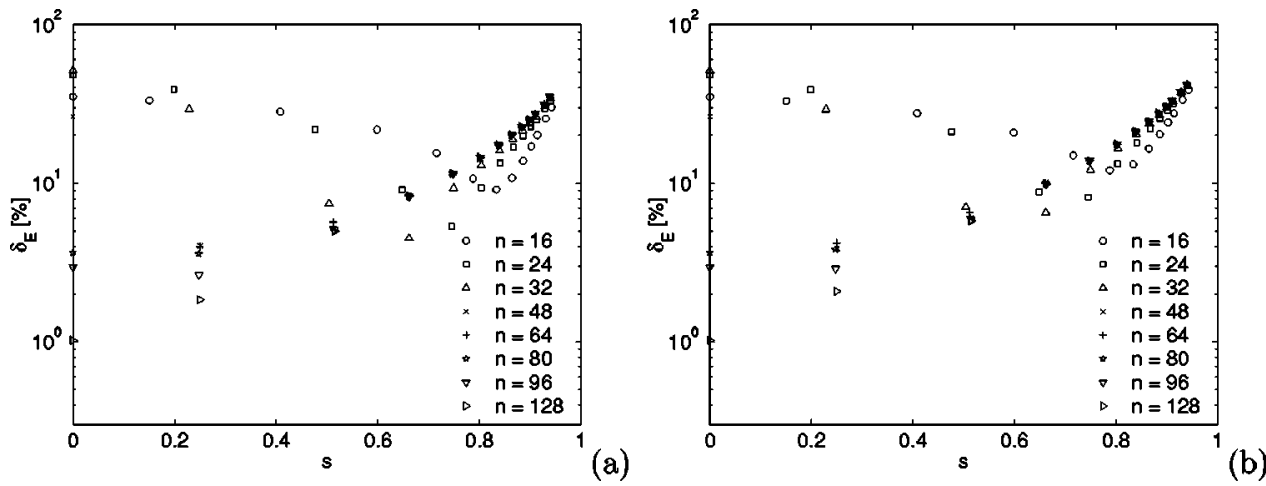

FIG. 10. Relative error $\delta_{E}$ (a) and $\hat{\delta}_{E}$ (b) for the kinetic energy at $\operatorname{Re}_{\lambda}=100$. 

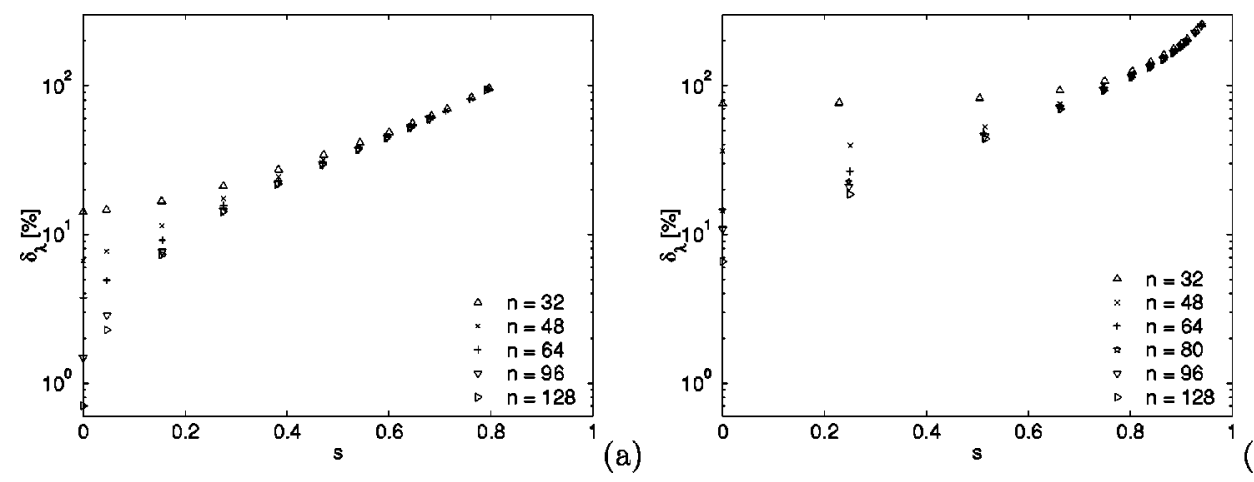

(b)

FIG. 11. Relative error $\hat{\delta}_{\lambda}$ for the Taylor length scale at $\operatorname{Re}_{\lambda}=50$ (a) and $\operatorname{Re}_{\lambda}=100$ (b).

depending on resolution and $s$. This arises from the interactions between modeling and discretization errors which were found to counteract. Operating LES near $s=s_{c}$ clearly provides a "safe" option for refining LES at a controlled level of discretization errors and consistent with an effective use of computational resources. Higher subgrid activities would yield a higher error, while lower values for $s$ do not guarantee an improvement in all cases. In Ref. 31 the dominance of the modeling errors for $s \gtrsim s_{c}$ was established. However, operating LES near $s=s_{c}$ may be sub-optimal with respect to realizing minimal total error at fixed computational cost. Lower error levels may be realized without increasing the computational cost, as a result of the counteracting balancing of the contributing errors. This will be discussed in Sec. V.

The same total error analysis can be performed for the Taylor length scale. The results for $\operatorname{Re}_{\lambda}=50$ and $\operatorname{Re}_{\lambda}=100$ are presented in Figs. 11(a) and 11(b), respectively. The results corresponding to $n=16$ and $n=24$ are not included in order to display the main trends more clearly. Here as well cross-over points can be distinguished at $s_{c} \approx 0.4$ and $s_{c}$ $\approx 0.8$ for $\operatorname{Re}_{\lambda}=50,100$, respectively. Although the error levels for $s \lesssim s_{c}$ show a strong dependence on the spatial resolution, the total error decreases monotonically for all resolutions considered. Hence, in contrast with the findings for the kinetic energy, an increased resolution is seen to provide a better prediction. This corresponds directly with the detailed error decomposition and the same sign of the error contributions presented in the previous section.

\section{ACCURACY CHARTS, OPTIMAL REFINEMENT STRATEGIES, AND COST ANALYSIS}

In the following, we introduce so-called "accuracy charts" in Sec. V A. These provide an effective means of quantifying the global error behavior as function of resolution and $C_{\mathrm{S}} \Delta$. Then, in Sec. V B, optimal refinement trajectories in the "resolution-filter width" space are defined. Further, the dependence of the accuracy charts and optimal refinement strategies on Reynolds number and subgrid activity will be quantified.

\section{A. Accuracy charts for the Smagorinsky model}

Instead of quantifying total errors against subgrid activity, a more detailed impression of the error behavior may be obtained by considering the total error as a function of the numerical resolution $n \sim 1 / h$ and effective subgrid resolution, defined as the ratio of the effective LES filter width $C_{S} \Delta$ and the grid-spacing $h$. From contour plots of $e_{\text {total }}$ or $\hat{e}_{\text {total }}$ an effective representation of the total errors in the individual large-eddy simulations can be obtained.

In Fig. 12(a), the total error contours for the kinetic energy are plotted as a function of $C_{S} \Delta / h$ and $n$ characterizing the different large-eddy simulations at $\operatorname{Re}_{\lambda}=50$. We will refer to this type of visualization as "accuracy charts." This accuracy chart allows one to interpret the effectiveness of different simulation strategies in reducing the total error. First of all, we may consider various simulations for which
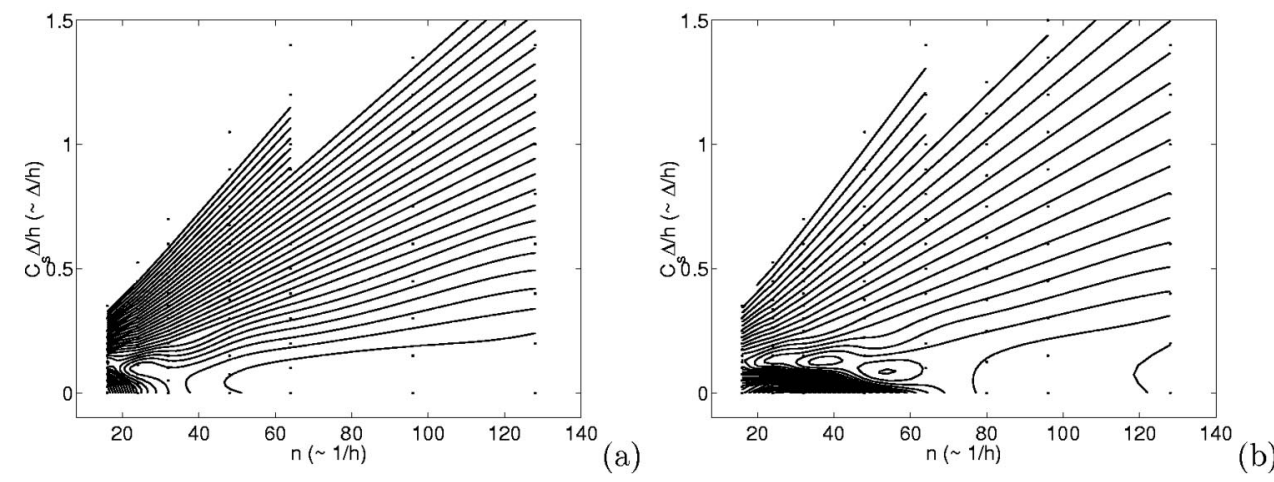

FIG. 12. Contours of $\hat{\delta}_{E}[\%]$ for the kinetic energy of LES at $\operatorname{Re}_{\lambda}=50$ (a) and $\operatorname{Re}_{\lambda}=100$ (b). Contour levels start at $1 \%$ (2\%) for the lower right contour and increase in steps of $1 \%(2 \%)$ for $\operatorname{Re}_{\lambda}=50\left(\operatorname{Re}_{\lambda}=100\right)$. Bullets correspond to locations of individual LES runs. 
$C_{\mathrm{S}} \Delta / h$ is kept constant while $n \sim 1 / h$ increases. Along these horizontal lines in Fig. 12, $C_{\mathrm{S}} \Delta$ decreases linearly with $h$. This corresponds to a popular choice in literature when refining large-eddy simulations. As $n$ increases the simulations range from coarse, significantly filtered LES over finer, filtered LES toward unfiltered DNS. The accuracy of the predictions will in most cases improve at an increasing computational cost which scales with $n^{4}$. Moving along vertical lines in the accuracy chart corresponds to changing the LES filter width at constant spatial resolution and thus at constant computational cost. At high values of $C_{\mathrm{S}} \Delta / h$ the turbulent flow is represented in a strongly smoothed way and the deviation from DNS data becomes quite unacceptable. A third important option for grid refinement arises from the choice to keep the filter-width $\Delta$ constant while refining the grid. At sufficiently high $n$ the numerical solution will approximate the grid independent solution to the modeled LES equations, in this case the Smagorinsky solution. Since $C_{\mathrm{S}} \Delta$ is constant in this case $C_{\mathrm{S}} \Delta / h$ is proportional to $n$. This refinement option corresponds to a set of straight lines through the origin with slopes $C_{S} \Delta$. Since moving along such refinement lines implies approaching a grid independent LES which is characterized by modeling error only, the error contour lines coincide with these straight lines in the grid independent LES region. This is clearly visible in the upper right corner of Fig. 12(a). We also considered the accuracy chart corresponding to $\delta_{E}$ instead of $\hat{\delta}_{E}$. The contour levels are slightly shifted in comparison to Fig. 12(a) but the main error pattern is unchanged. Likewise, in Fig. 12(b) $\hat{\delta}_{E}$ is presented for the $\operatorname{Re}_{\lambda}=100$ case. Contour levels are considerably higher than in the low Reynolds number case. However, the primary error pattern of both Reynolds number cases is remarkably similar.

To summarize, the accuracy charts for the kinetic energy exhibit the same patterns, quite independent of the error definition and with only a gradual dependence on the Reynolds number. Hence, these charts appear as a "fingerprint" for the accuracy with which a flow quantity can be predicted by a specific LES system, i.e., the combination of a numerical method and subgrid model. It gives an overview of the error behavior under all sorts of numerical and physical parameter settings and provides a useful tool to quantify and compare the quality of different numerical methods and subgrid models. Further, accuracy charts provide an easy way to determine optimal working conditions for a LES system balancing optimal accuracy with computational cost.

In Fig. 13, the accuracy chart for the Taylor length scale predictions is presented at $\operatorname{Re}_{\lambda}=100$. Here, the observed pattern differs significantly from that of $\delta_{E}$, which is due to the different nature of the interaction between modeling and discretization errors. Basically, the same observation was obtained in Sec. IV, where these errors were studied as function of the subgrid activity.

In Sec. V B we will use the accuracy-chart information to determine optimal refinement strategies.

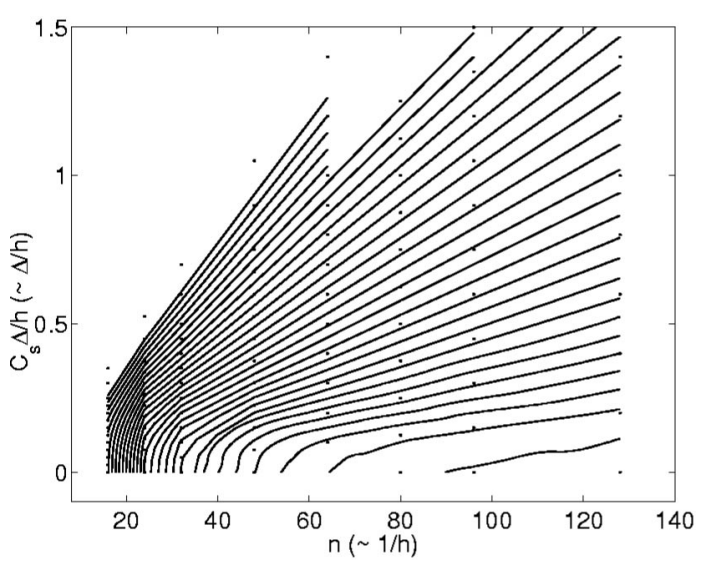

FIG. 13. Contours of $\hat{\delta}_{\lambda}[\%]$ for the Taylor length scale of different LES at $\operatorname{Re}_{\lambda}=100$. Contour levels start at $12 \%$ for the lower right contour and increase in steps of $8 \%$. Bullets correspond to locations of different LES runs.

\section{B. Optimal refinement strategies}

Based on the information contained in an accuracy chart, an optimal refinement strategy for a variable $\phi$ can be determined. Consider for this purpose the error behavior expressed by $\delta_{\phi}(n, \Delta / h)$. The optimal accuracy for a given resolution $n$ is obtained as

$$
\delta_{\phi, \mathrm{opt}}(n)=\min _{C_{\mathrm{S}} \Delta / h}\left[\delta_{\phi}\left(n, \frac{C_{\mathrm{S}} \Delta}{h}\right)\right] .
$$

This minimum defines the optimal effective subgrid resolution $\left(C_{\mathrm{S}} \Delta / h\right)_{\text {opt }}$ as a function of resolution and the optimal accuracy trajectory is defined as the curve $\left\{n,\left(C_{\mathrm{S}} \Delta / h\right)_{\text {opt }}\right\}$. For every $n$ the minimal error arises from the proper balancing between modeling and discretization errors.

In Fig. 14 the optimal trajectory for the kinetic energy is plotted on the $\hat{\delta}_{E}$ chart for $\operatorname{Re}_{\lambda}=50$ and $\operatorname{Re}_{\lambda}=100$. From a detailed error analysis (cf. Sec. III A) in the vicinity of the optimal trajectory it is found that the modeling and discretization error effects maximally balance each other. As an example the optimal point for a $32^{3}$ grid and $\operatorname{Re}_{\lambda}=100$ is situated between $C_{\mathrm{S}} \Delta=0.1 h$ and $C_{\mathrm{S}} \Delta=0.2 h$, which were discussed in detail in Sec. III. In case $C_{\mathrm{S}} \Delta$ is increased at constant $n$ in the region above the optimal trajectory, the modeling error will become dominant. In case $C_{\mathrm{S}} \Delta$ is decreased below the optimal trajectory, the numerical error will dominate the total error. Especially at coarser resolutions the corresponding increase in total error is very large. In Sec. IV, $s \approx s_{c}$ was quantified as a "safe" region to operate LES. Therefore, the $s=s_{c}$ trajectory is also displayed in Fig. 14. It can clearly be seen that a gain in accuracy can be obtained at constant computational cost when $C_{\mathrm{S}} \Delta$ is decreased from the $s=s_{c}$ condition toward the optimal trajectory. This is particularly relevant at high resolutions which allow an optimal setting close to DNS. However, it is also clear that decreasing $C_{S} \Delta$ too much, and hence dropping below the optimal trajectory, can yield a spectacular increase in error. This is especially relevant at low resolutions in the lower left of the accuracy chart. This specific region is rather small for $\mathrm{Re}_{\lambda}$ $=50$, but increases for $\operatorname{Re}_{\lambda}=100$. 

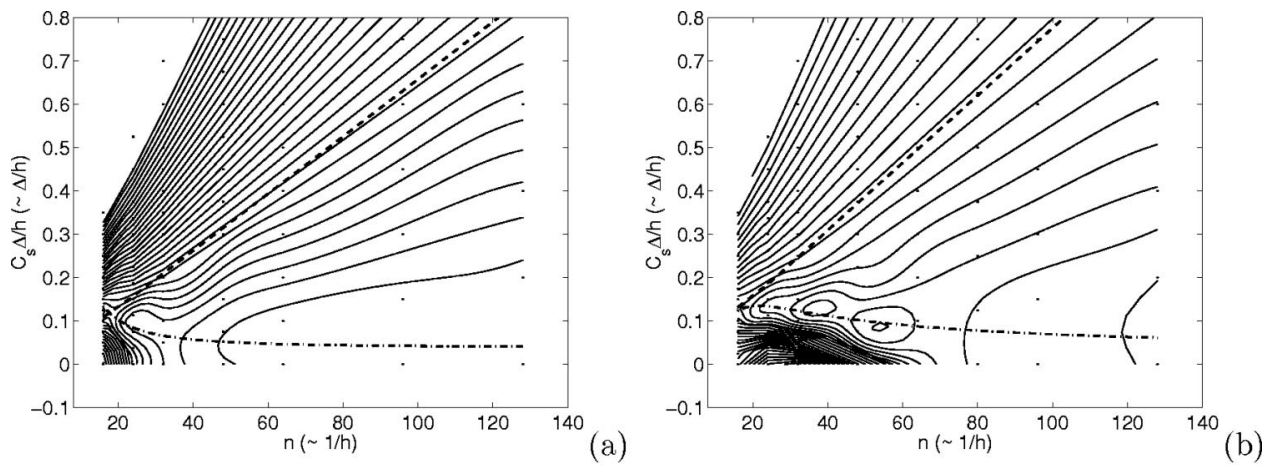

FIG. 14. Optimal trajectory and accuracy chart based on $\hat{\delta}_{E}[\%]$ for $\operatorname{Re}_{\lambda}=50$ (a) and $\operatorname{Re}_{\lambda}=100$ (b). Optimal refinement trajectory (-.-); line with $s=s_{c}$ (---) with $s_{c}=0.4$ in (a) and $s_{c}=0.8$ in (b). Contour levels start at $1 \%(2 \%)$ at the lower right contour and increase in steps of $1 \%(2 \%)$ for $\operatorname{Re}_{\lambda}=50$ ( $\operatorname{Re}_{\lambda}$ $=100)$.

In previous sections the error patterns and accuracy charts based on the Taylor length scale were found to exhibit important differences compared to errors based on the kinetic energy. This was associated with differences in the interaction between the basic error components. If an optimal refinement trajectory would be based on minimal $\delta_{\lambda}(n, \Delta / h)$, this optimal trajectory would obviously differ from that based on kinetic energy errors. In Fig. 15, the accuracy charts based on $\hat{\delta}_{\lambda}$ for $\operatorname{Re}_{\lambda}=50$ and $\operatorname{Re}_{\lambda}=100$ are presented. In addition, the optimal trajectories based on $\hat{\delta}_{E}$ are displayed. As can be seen in both charts, the error in $\lambda$ is minimal at $C_{\mathrm{S}} \Delta=0.0$, i.e., for the under-resolved DNS setting. However, at small to medium resolutions, the error contours are perpendicular to the coordinate axis, which indicates that the location of the optimum is not sharply defined and a region in the vicinity of $C_{\mathrm{S}} \Delta=0$ may serve to provide acceptable near-optimal parameter settings. The optimal refinement trajectories based on $\hat{\delta}_{E}$ appear not too far off for $\lambda$. Moreover, by the very nature of large-eddy simulation one may put a larger emphasis on capturing flow quantities associated with the larger retained scales, such as the kinetic energy, compared to inertial range properties such as the Taylor length scale. This is particularly true in relation to the Smagorinsky model, which is known to provide too much dissipation in the smaller retained scales and hence distorts the prediction of these quantities much more than occurs for the larger retained scales.

Finally, we turn to the optimal refinement strategies and summarize the influence of the specific error definitions on the optimal refinement strategy in Fig. 16(a). As can be seen, the lines based on $\delta_{E}$ are shifted upward slightly compared to those based on $\hat{\delta}_{E}$. For $\operatorname{Re}_{\lambda}=50$, the difference is marginal. However, for $\operatorname{Re}_{\lambda}=100$, a maximum shift of $10 \%$ is observed. The difference induced by both error definitions gives rise to an uncertainty in the optimal trajectory. It is seen that one obtains an "optimal refinement band" shaded gray in this figure. In Fig. 16(b), the optimal refinement strategies (with uncertainty bands) are plotted as function of the relative subgrid activity $s / s_{c}$. For $\operatorname{Re}_{\lambda}=100$, it is interesting to notice that the optimal trajectory at low resolutions is at constant subgrid activity $s / s_{c} \approx 1$. Hence, in this region the subgrid model dominates the dissipation rate in the LES. At higher resolutions, starting from $n \approx 48$, the optimal trajectory evolves toward $s=0$ according to a power law $\left(\sim n^{-3 / 2}\right)$. In case the Smagorinsky model is used it hence appears better to increase the resolution and decrease the importance of the subgrid fluxes if one tries to obtain accurate predictions. There is no such quasi-constant region at $\operatorname{Re}_{\lambda}=50$. Here, the optimal trajectory at lowest resolution also starts at $s / s_{c} \approx 1$ and the power law decay $\left(\sim n^{-3}\right)$ to-
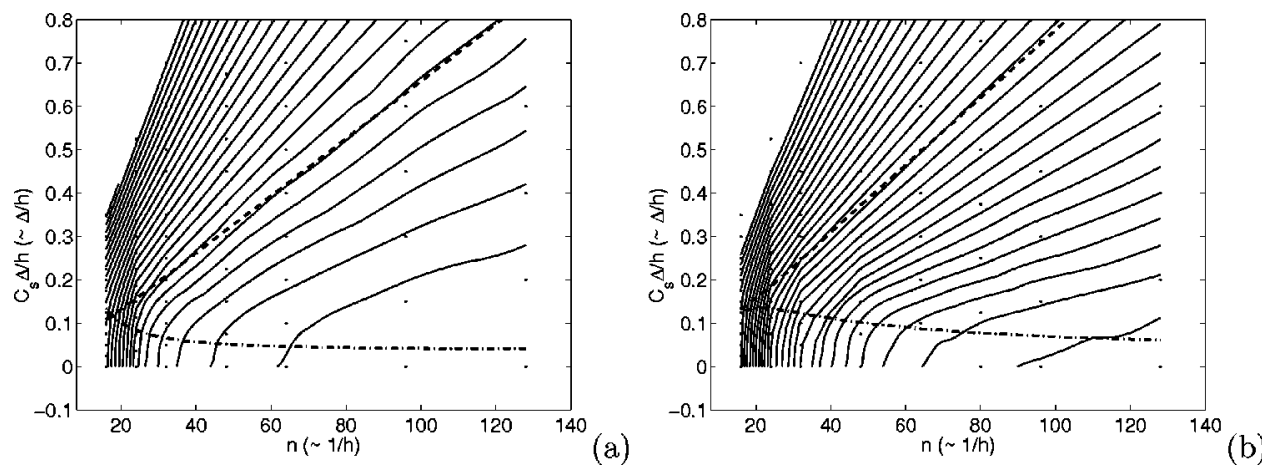

FIG. 15. Optimal refinement strategy based on $\hat{\delta}_{E}$ and accuracy chart based on $\hat{\delta}_{\lambda}[\%]$ for $\operatorname{Re}_{\lambda}=50$ (a) and $\operatorname{Re}_{\lambda}=100$ (b). Optimal refinement trajectory (-.-); line at $s=s_{c}$ (---) with $s_{c}=0.4$ in (a) and $s_{c}=0.8$ in (b). Contour levels start at $4 \%(12 \%)$ at the lower right contour and increase in steps of $4 \%$ (8\%) for $\operatorname{Re}_{\lambda}=50\left(\operatorname{Re}_{\lambda}=100\right)$. 

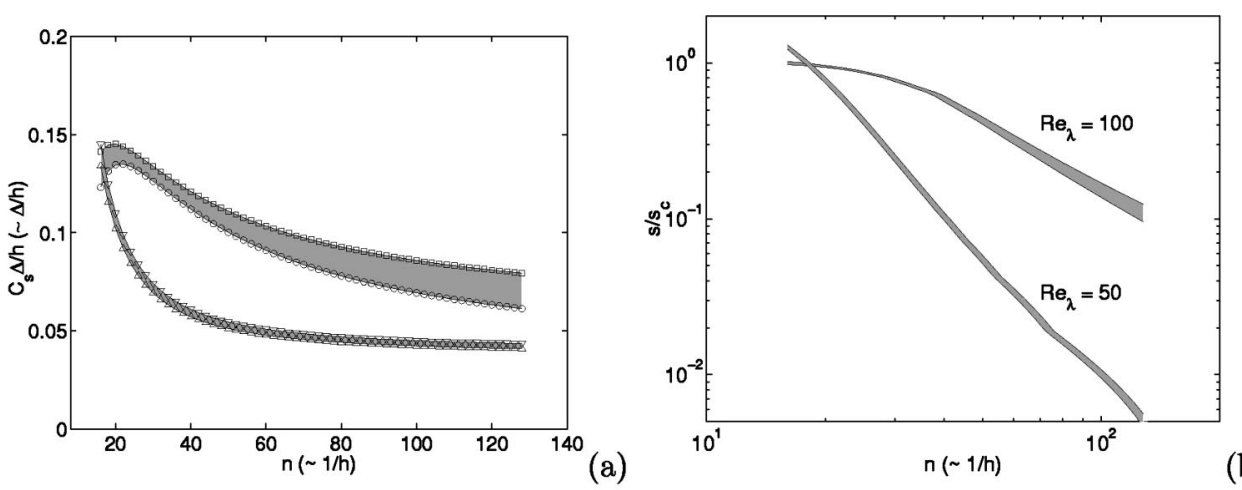

(b)

FIG. 16. (a) Optimal refinement strategy for different Reynolds numbers and error definitions: ( $\square$ ) based on $\delta_{E}$ at $\operatorname{Re}_{\lambda}=100 ;(\bigcirc) \hat{\delta}_{E}$ at $\operatorname{Re}_{\lambda}=100$; $(\nabla) \delta_{E}$ at $\operatorname{Re}_{\lambda}=50 ;(\triangle) \hat{\delta}_{E}$ at $\operatorname{Re}_{\lambda}=100$. (b) Optimal refinement strategies for $\operatorname{Re}_{\lambda}=50$ and $\operatorname{Re}_{\lambda}=100$ as function of the relative subgrid activity $s / s_{c}$, where $s_{c}=0.4$ at $\operatorname{Re}_{\lambda}=50$ and 0.8 at $\operatorname{Re}_{\lambda}=100$, respectively.

ward no model LES arises directly. An extension of the database to include other, higher, Reynolds numbers is needed to classify whether this dependence of the power law exponent on Reynolds number and the cross-over point scaling are indicative of more general error behavior.

\section{CONCLUDING REMARKS}

An extensive database was set up for the large-eddy simulation of homogeneous isotropic turbulence. This database consists of two reference direct numerical simulations at two different initial Taylor Reynolds numbers, i.e., 50 and 100. Further, large-eddy simulations, using a Smagorinsky subgrid model, were performed at different resolutions $n$ and subgrid resolutions $\Delta / h$ for both Reynolds numbers. This allows one to obtain detailed information on the error behavior, i.e., total, modeling as well as discretization errors, as function of relevant physical and numerical parameters.

A detailed analysis of the different error contributions was discussed. For the error in the total kinetic energy it was observed that modeling and discretization errors may partially cancel each other. Correspondingly, the total error can be lower than its contributing parts. Depending on the specific subgrid resolution, this error interaction can be more or less balanced. Similar effects were observed for the total kinetic energy and the momentum thickness in a simulation of a temporal mixing layer. ${ }^{29}$ It is tempting to hypothesize that the error balancing may be effective in more general situations. Further simulations of other, canonical flows is required to assess this. When the same analysis was performed for the Taylor length scale it was found that numerical and modeling error effects have the same sign.

A global error analysis and classification of errors was performed, mainly based on the $L_{2}$ norm of the total error. We observed that an error definition with reference to a filtered DNS provides similar error patterns compared to an error definition that is based on unfiltered DNS data. Hence, conclusions pertaining to optimal simulation strategies may be globally inferred independent of the specific error definitions.

First of all, the total errors of the large-eddy simulations as a function of the subgrid activity $s$ were evaluated. Visu- ally, a cross-over point $s_{c}$ can be identified, which divides the error behavior into two main regions. This cross-over point depends on the Reynolds number and is situated at $s \approx 0.4$ and $s \approx 0.8$ for $\operatorname{Re}_{\lambda}=50$ and $\operatorname{Re}_{\lambda}=100$, respectively. For $s$ $\gtrsim s_{c}$ the total error is dominated by modeling error effects and, roughly speaking, is a function of $s$ only. The minimal error in this region is situated at $s_{c}$. For $s \lesssim s_{c}$ errors are scattered considerably and dominated by discretization error effects.

When an overview of errors is considered in the $\left\{n, C_{s} \Delta / h\right\}$ plane, the full error behavior may be concisely visualized. It is straightforward to obtain the optimal working conditions for large-eddy simulations from such accuracy charts. These optimal trajectories were based on error levels of the kinetic energy. It is shown that the optimal refining trajectory differs considerably from the $s \approx s_{c}$ trajectory. At high resolution, the latter corresponds to approximating the grid independent Smagorinsky solution which approaches a constant error level. Certainly for higher resolutions, the difference in accuracy is substantial and the optimal trajectory approaches a DNS setting at high resolutions. These high resolutions are, however, of limited practical relevance in view of the desire in LES to significantly reduce computational costs. For the latter, the findings at low resolutions are more meaningful in the context of LES at very high Reynolds numbers.

When the optimal LES trajectories were plotted against the subgrid activity, a decreasing power law relation between $s / s_{c}$ and $n$ was established. Hence, at high resolutions the optimal trajectory approaches the no-model LES at an algebraic rate for the Smagorinsky model. The exponent for $\operatorname{Re}_{\lambda}=50$ is twice that of the $\operatorname{Re}_{\lambda}=100$ case. Further, for both Reynolds numbers, the optimal trajectories start for low resolutions approximately at $s_{c}$, i.e., at coarse grids optimal accuracy is consistent with constant subgrid activity, especially at high Reynolds numbers.

The relevance of this database approach for different subgrid models and different spatial discretization methods is presently under investigation. From a collection of such database analyses for various "building-block" flows it is expected that one may extract guidelines for determining $a$ 
priori numerical and physical parameter settings which allow "near-optimal" large-eddy simulations for general, practically relevant turbulent flow problems. The present study provides a first step toward this goal.

\section{ACKNOWLEDGMENTS}

This research was performed in the framework of FWOproject No. G.0130.02. The authors acknowledge the financial support by FWO-Vlaanderen and further acknowledge support from NCF (National Computing Foundation) to make the extensive simulations possible.

${ }^{1}$ A. N. Kolmogorov, "The local structure of turbulence in an incompressible viscous fluid with very large Reynolds numbers," Proc. R. Soc. London, Ser. A 434, 9 (1991) [first published in Dokl. Akad. Nauk SSSR 30, 301 (1941)].

${ }^{2}$ J. Smagorinsky, "General circulation experiments with the primitive equations," Mon. Weather Rev. 91, 99 (1963).

${ }^{3}$ U. Schumann, "Subgrid scale model for finite difference simulations of turbulent flows in plane channels and annuli," J. Comput. Phys. 18, 376 (1975).

${ }^{4}$ R. H. Kraichman, "Eddy viscosity in two and three dimensions," J. Atmos. Sci. 33, 1512 (1976).

${ }^{5} \mathrm{O}$. Métais and M. Lesieur, "Spectral large-eddy simulation of isotropic and stably stratified turbulence," J. Fluid Mech. 293, 157 (1992).

${ }^{6} \mathrm{C}$. E. Leith, "Stochastic backscatter in a subgrid-scale model: Plane shear mixing layer," Phys. Fluids A 2, 297 (1990).

${ }^{7}$ A. Yoshizawa, "A statistically-derived subgrid model for the large-eddy simulation of turbulence," Phys. Fluids 25, 1532 (1982).

${ }^{8}$ R. A. Clark, J. H. Ferziger, and W. Reynolds, "Evaluation of subgrid-scale models using an accurately simulated turbulent flow," J. Fluid Mech. 91, 1 (1979).

${ }^{9}$ J. Bardina, J. H. Ferziger, and W. C. Reynolds, "Improved subgrid scale models for large eddy simulation," in AIAA 13th Fluid \& Plasma Dynamics Conference, Snowmass, CO, 1980.

${ }^{10}$ A. Leonard, "Large-eddy simulation of chaotic convection and beyond," Tech. Rep. 97-0204, AIAA (1997).

${ }^{11}$ B. J. Geurts, "Inverse modeling for large-eddy simulation," Phys. Fluids 9, 3585 (1997).

${ }^{12} \mathrm{~K}$. Horiuti, "A new dynamic two-parameter mixed model for large-eddy simulation," Phys. Fluids 9, 3443 (1997).

${ }^{13}$ G. S. Winckelmans, A. A. Wray, and O. V. Vasilyev, "Testing of a new mixed model for LES: The Leonard model supplemented by a dynamic Smagorinsky term," Proceedings of the Summer Program 1998 (Center for Turbulence Research, Stanford, 1998), pp. 367-388.

${ }^{14}$ A. Leonard and G. S. Winckelmans, "A tensor-diffusivity subgrid model for large-eddy simulation," in Direct and Large-Eddy Simulation III, edited by P. R. Voke, N. D. Sandham, and L. Kleiser (Kluwer Academic, Dordrecht, 1999), pp. 147-162.

${ }^{15}$ Y. Zang, R. L. Street, and J. R. Koseff, "A dynamic mixed subgrid-scale model and its application to turbulent recirculating flows," Phys. Fluids A 5, 3186 (1993)

${ }^{16}$ B. Vreman, B. J. Geurts, and H. Kuerten, "On the formulation of the dynamic mixed subgrid-scale model," Phys. Fluids 6, 4057 (1994)

${ }^{17}$ S. Stolz and N. A. Adams, "An approximate deconvolution procedure for large-eddy simulation,” Phys. Fluids 11, 1699 (1999).

${ }^{18}$ B. J. Geurts, I. C. C. de Bruin, and S. Sarkar, "Rapid and slow contributions to the turbulent stress tensor and inverse modeling in a turbulent mixing layer," in Advances in Turbulence VIII. Proceedings of the Eighth European Turbulence Conference, edited by C. Dopazo, 2000, pp. 539542.

${ }^{19}$ J. Meyers, M. Baelmans, and C. Lacor, "A generalised view on mixed models," in Advances in Turbulence VIII. Proceedings of the Eighth European Turbulence Conference, edited by C. Dopazo, 2000, pp. 515-518.

${ }^{20}$ J. A. Domaradzki and E. M. Saiki, "A subgrid-scale model based on the estimation of unresolved scales of turbulence," Phys. Fluids 9, 2148 (1997).

${ }^{21}$ J. A. Domaradzki and P. P. Yee, "The subgrid-scale estimation model for high Reynolds number turbulence," Phys. Fluids 12, 193 (2000).

${ }^{22}$ J. A. Domaradzki and K.-C. Loh, "The subgrid-scale estimation model in the physical space representation," Phys. Fluids 11, 2330 (1999).

${ }^{23}$ A. Scotti and C. Meneveau, "A fractal model for large eddy simulation of turbulent flow," Physica D 127, 198 (1999).

${ }^{24}$ B. J. Geurts and D. D. Holm, "Regularization modeling for large-eddy simulation," Phys. Fluids 15, L13 (2003).

${ }^{25}$ M. Germano, U. Piomelli, P. Moin, and W. H. Cabot, "A dynamic subgridscale eddy viscosity model," Phys. Fluids A 3, 3128 (1991).

${ }^{26}$ M. Germano, "Turbulence: The filtering approach,” J. Fluid Mech. 238, 325 (1992)

${ }^{27}$ D. K. Lilly, "A proposed modification of the Germano subgrid-scale closure method," Phys. Fluids A 4, 633 (1992).

${ }^{28} \mathrm{~S}$. Ghosal, "An analysis of numerical errors in large-eddy simulations of turbulence," J. Comput. Phys. 125, 187 (1996).

${ }^{29}$ B. Vreman, B. Geurts, and H. Kuerten, "Comparison of numerical schemes in large-eddy simulations of the temporal mixing layer," Int. J. Numer. Methods Fluids 22, 297 (1996).

${ }^{30}$ B. J. Geurts, "Balancing errors in LES," in Direct and Large-Eddy Simulation III, Ercoftac Series Vol. 7, edited by P. R. Voke and N. D. Sandham (Kluwer Academic, Dordrecht, 1999).

${ }^{31}$ B. J. Geurts and J. Fröhlich, "A framework for predicting accuracy limitations in large eddy simulations," Phys. Fluids 14, L41 (2002).

${ }^{32} \mathrm{~S}$. Ghosal, "Analysis and control of errors in the numerical simulation of turbulence," in Turbulent Flow Computation, edited by D. Drikakis and B. J. Geurts (Kluwer Academic, Dordrecht, 2002), pp. 101-141.

${ }^{33}$ B. Vreman, B. Guerts, and H. Kuerten, "Large-eddy simulation of the turbulent mixing layer," J. Fluid Mech. 339, 357 (1997).

${ }^{34} \mathrm{~W}$. Ijzerman, "Signal representation and modeling of spatial structures in fluids," Ph.D. thesis, Universiteit Twente, 2000.

${ }^{35}$ S. B. Pope, Turbulent Flows (Cambridge University Press, Cambridge, 2000).

${ }^{36}$ S. Tavoularis, J. C. Bennett, and S. Corrsin, "Velocity-derivative skewness in small Reynolds number, nearly isotropic turbulence," J. Fluid Mech. 88, 63 (1978).

${ }^{37}$ G. K. Batchelor, The Theory of Homogeneous Turbulence, Cambridge Monographs on Mechanics and Applied Mathematics (Cambridge University Press, Cambridge, 1953).

${ }^{38}$ J. W. Deardoff, "A numerical study of three-dimensional turbulent channel flow at large Reynolds numbers," J. Fluid Mech. 41, 453 (1970).

${ }^{39}$ O. J. McMillan and J. H. Ferziger, "Direct testing of subgrid-scale models," AIAA J. 17, 1340 (1979).

${ }^{40}$ D. K. Lilly, in Proceedings of IBM Scientific Computing Symposium on Environmental Sciences, IBM Data Processing Division, White Plains, NY, 1967.

${ }^{41}$ B. Vreman, B. J. Geurts, and H. Kuerten, "Realizability conditions for the turbulent stress tensor in large-eddy simulation,” J. Fluid Mech. 278, 351 (1994).

${ }^{42}$ A. Muschinski, "A similarity theory of locally homogeneous and isotropic turbulence generated by a Smagorinsky-type LES," J. Fluid Mech. 325, 239 (1996). 
Physics of Fluids is copyrighted by the American Institute of Physics (AIP). Redistribution of journal material is subject to the AIP online journal license and/or AIP copyright. For more information, see http:/ojps.aip.org/phf/phfcr.jsp

Copyright of Physics of Fluids is the property of American Institute of Physics and its content may not be copied or emailed to multiple sites or posted to a listserv without the copyright holder's express written permission. However, users may print, download, or email articles for individual use. 